\title{
Antiinflammatory activity of ANGPTL4 facilitates macrophage polarization to induce cardiac repair
}

\author{
Dong Im Cho, ${ }^{1}$ Hye-jin Kang, ${ }^{1}$ Ju Hee Jeon, ${ }^{1}$ Gwang Hyeon Eom, ${ }^{2}$ Hyang Hee Cho, ${ }^{1,3}$ Mi Ra Kim, ${ }^{1}$ \\ Meeyoung Cho, ${ }^{1}$ Hye-yun Jeong, ${ }^{1}$ Hyen Chung Cho, ${ }^{1,3}$ Moon Hwa Hong, ${ }^{1}$ Yong Sook Kim, ${ }^{1,4}$ \\ and Youngkeun Ahn ${ }^{1,5}$ \\ ${ }^{1}$ Cell Regeneration Research Center, Chonnam National University Hospital, Gwangju, Korea. ${ }^{2}$ Department of \\ Pharmacology, Chonnam National University Medical School, Gwangju, Korea. ${ }^{3}$ Department of Molecular Medicine, \\ Graduate School, Chonnam National University, Gwangju, Korea. ${ }^{4 B i o m e d i c a l ~ R e s e a r c h ~ I n s t i t u t e, ~ C h o n n a m ~ N a t i o n a l ~}$ \\ University Hospital, Gwangju, Korea. ${ }^{5}$ Department of Cardiology, Chonnam National University Medical School, \\ Gwangju, Korea.
}

Mesenchymal stem cells (MSCs) can suppress pathological inflammation. However, the mechanisms underlying the association between MSCs and inflammation remain unclear. Under coculture conditions with macrophages, MSCs highly expressed angiopoietin-like 4 (ANGPTL4) to blunt the polarization of macrophages toward the proinflammatory phenotype. ANGPTL4-deficient MSCs failed to inhibit the inflammatory macrophage phenotype. In inflammation-related animal models, the injection of coculture medium or ANGPTL4 protein increased the antiinflammatory macrophages in both peritonitis and myocardial infarction. In particular, cardiac function and pathology were markedly improved by ANGPTL4 treatment. We found that retinoic acid-related orphan receptor $\alpha(R O R \alpha)$ was increased by inflammatory mediators, such as IL-1 $\beta$, and bound to ANGPTL4 promoter in MSCs. Collectively, ROR $\alpha$-mediated ANGPTL4 induction was shown to contribute to the antiinflammatory activity of MSCs against macrophages under pathological conditions. This study suggests that the capability of ANCPTL4 to induce tissue repair is a promising opportunity for safe stem cell-free regeneration therapy from a translational perspective.

Conflict of interest: The authors have declared that no conflict of interest exists.

Copyright: () 2019, American Society for Clinical Investigation.

Submitted: October 10, 2018

Accepted: July 23, 2019

Published: August 22, 2019

Reference information: /CI Insight. 2019:4(16):e125437.

https://doi.org/10.1172/jici.

insight.125437.

\section{Introduction}

Mesenchymal stem cell-mediated (MSC-mediated) regeneration therapy has been widely applied for cardiovascular disease (1-4). MSCs effect tissue repair largely via their paracrine factors and by activating endogenous progenitor cells, not only by cell replacement $(1,5,6)$. Inflammation is a normal response to pathological stress, and chronic degenerative disorders are usually associated with uncontrolled inflammation.

Unlike the well-characterized paracrine effects and differentiation potential of MSCs, the contributing mediators against inflammatory macrophages within the context of pathological progress remain to be established. We and others have shown that MSCs can modulate the macrophage phenotype; however, the underlying mechanism has remained unclear to date (7). Macrophages are actively involved in pathological changes. In the early phase, circulating monocytes infiltrate damaged tissue and differentiate into macrophages to participate in phagocytosis with proinflammatory properties (classic M1 phenotype). M1 macrophages produce proinflammatory mediators, such as nitric oxide. In the late phase, alternatively activated M2-type macrophages induce the resolution of inflammation and tissue regeneration. When this normal process is not controlled, chronic inflammation leads to functional decline without sufficient repair. A delicate regulation of macrophage phenotype is thus functionally relevant to tissue repair, and we previously showed that chemical-induced macrophage modulation can significantly contribute to cardiac recovery after myocardial infarction (MI) (8-10).

MSCs migrate to sites of tissue injury; however, most MSCs are cleared. Despite these limitations, MSCs continue to yield therapeutic benefits in a large number of disease models. MSCs produce and release abundant growth factors and cytokines and recently have been reported to secrete exosomes, which are rich in nucleic acids, proteins, and miRNA. Intercellular cross-talk with MSCs is highly sophisticated and involves multiple modes of communication to maintain homeostasis in the microenvironment. 
In this study, we investigated whether MSC-derived mediators act during inflammation to control the progression of pathological changes. We found that MSCs highly induced angiopoietin-like 4 (ANGPTL4) to suppress activation of inflammatory macrophages. ANGPTL4 is a secreted angiopoietin-like glycoprotein and is a definite factor for commitment to antiinflammatory macrophages. ANGPTL4 is also known to play an important role in controlling innate immune responses. Given the critical role of ANGPTL4 in MSCs, we set out to study the physiological relevance of MSC-derived ANGPTL4 in mouse models of peritonitis and MI.

\section{Results}

Human MSCs inhibit inflammatory activation of macrophages via ANGPTL4. In this study, we used cells as summarized in Supplemental Figure 1; supplemental material available online with this article; https://doi. org/10.1172/jci.insight.125437DS1. MSCs originated from human bone marrow (hMSCs), human adipose tissue (hATSCs), or mouse bone marrow (mMSCs). Macrophage cells were derived from human monocytic THP1 cells, human circulating CD14+ monocytes (Supplemental Figure 2), or mouse bone marrow cells (BMDMs). Thioglycollate elicited peritoneal macrophages (pMFs) and cardiac macrophages were harvested from peritonitis and MI mouse models, respectively. Using Transwell plates for coculture, we seeded MSCs or macrophages in the lower plate for further analysis. Among the highest ranked genes, we selected Angptl4 (Supplemental Table 1) as a responsible factor for the antiinflammatory effect on inflammatory macrophages. We showed that ANGPTL4 mRNA and protein were consistently increased in hMSCs cocultured with LPS-stimulated human $\mathrm{CD}_{14}^{+}$monocyte-derived macrophages (hMFs) (Figure 1A). Given that ANGPTL4 is highly upregulated in MSCs when cocultured with macrophages, we sought to determine the role of ANGPTL4. We performed a successful knockdown of ANGPTL4 in hMSCs by using siRNA (Supplemental Figure 3, A-C). After ANGPTL4 knockdown, the inhibitory activity of hMSCs on inflammatory CXCL10 in THP-1 macrophages (Supplemental Figure 3D) and inflammatory genes (Cxcl9, Cxcl10, $C x c l 11, C c r 7,1 l 6$, and $C c l 2)$ in hMFs (Figure 1B) was impaired. Because ANGPTL4 is a released protein, we sought to determine whether ANGPTL4 itself was directly associated with antiinflammatory activity of MSCs. Treatment of LPS-stimulated hMFs with recombinant ANGPTL4 protein attenuated the induction of proinflammatory genes in hMFs (Figure 1C).

Next, we treated human monocytic THP-1 cells with phorbol 12-myristate 13-acetate (PMA) to differentiate the cells to macrophages, and we replaced hMFs with differentiated THP-1 macrophages. Proinflammatory CXCL10 mRNA and protein were significantly reduced by coculture with hMSCs in LPS-stimulated THP-1 macrophages (Figure 1D). PMA-differentiated THP-1 macrophages also exhibited downregulation of inflammatory mediators by coculture with hMSCs, and we used THP-1 macrophages for mechanistic studies. To ensure that the aforementioned observations were not limited to hMSCs, we also used hATSCs and found that ANGPTL4 was highly upregulated in hATSCs cocultured with LPS-stimulated THP-1 macrophages (Supplemental Figure 3E). These results demonstrated that MSC-released ANGPTL4 was required to exert the antiinflammatory effect. Next we examined the involvement of the NF- $\mathrm{KB}$ pathway by measuring the degradation of $\mathrm{I} \kappa \mathrm{B} \alpha$, an endogenous inhibitor of $\mathrm{NF}-\kappa \mathrm{B}$, in macrophages. The typical degradation of $\mathrm{I} \kappa \mathrm{B} \alpha$ followed by restoration of the protein was observed in response to LPS, whereas I $\mathrm{K} B \alpha$ protein levels were not changed substantially in macrophages cocultured with hMSCs (Figure 1E). These results showed that hMSC coculture attenuated the proinflammatory responses via NF- $\mathrm{B}$ inhibition in LPS-stimulated macrophages. Using hMFs and THP-1 macrophages, we confirmed that the inflammatory macrophages highly induced ANGPTL4 in hMSCs. Additionally, we noted that ANGPTL4 was upregulated in hMSCs by coculture with macrophages regardless of LPS stimulation (Figure 1, F-H). LPS-induced proinflammatory genes were reduced by recombinant ANGPTL4 treatment dose dependently in THP-1 macrophages as observed in hMFs (Figure 1I). Thus, we used THP-1 macrophages for further studies.

ANGPTL4 is essential for an antiinflammatory effect on macrophages in MSCs. To examine the involvement of ANGPTL4 in the antiinflammatory activity of MSCs, we isolated mMSCs from ANGPTL4-knockout mice (Supplemental Figure 4). LPS-stimulated BMDMs were cocultured with either wild-type mMSCs or knockout mMSCs for 24 hours or 48 hours, and inflammation-related genes were analyzed. Antiinflammatory activity was shown only in wild-type mMSCs and not in knockout mMSCs (Figure 2A). In the same setting, induction of proinflammatory iNOS protein was prominently blocked, and antiinflammatory Arg1 protein was significantly upregulated in LPS-stimulated BMDMs by coculture with wild-type mMSCs but not with knockout mMSCs (Figure 2B). These results suggested that ANGPTL4 in MSCs is critical for exerting the antiinflammatory effect against macrophages. 
A
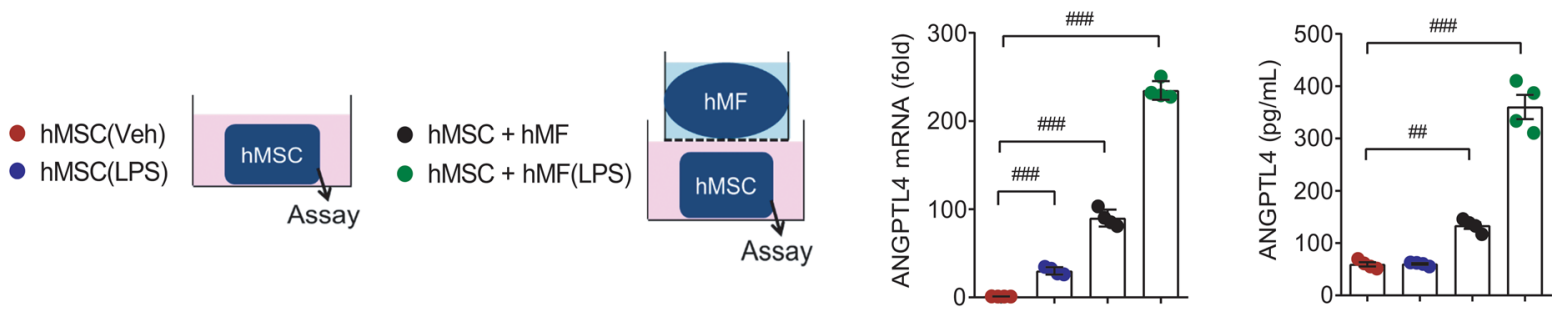

B

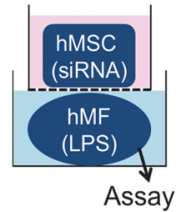

- hMF(LPS)

- $h M F(L P S)+h M S C($ siCon)

- hMF(LPS) + hMSC(siANGPTL4)
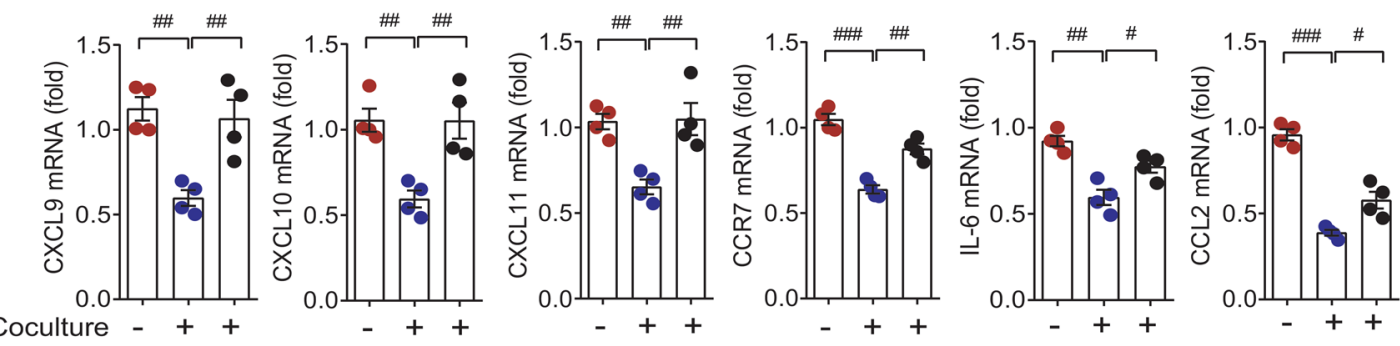

C

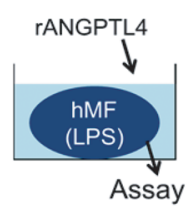

- $\mathrm{hMF}(\mathrm{LPS})+$ Veh

- $\mathrm{hMF}(\mathrm{LPS})+0.1 \mu \mathrm{g} / \mathrm{mL}$ rANGPTL4

- $\mathrm{hMF}(\mathrm{LPS})+1 \mu \mathrm{g} / \mathrm{mL}$ rANGPTL4
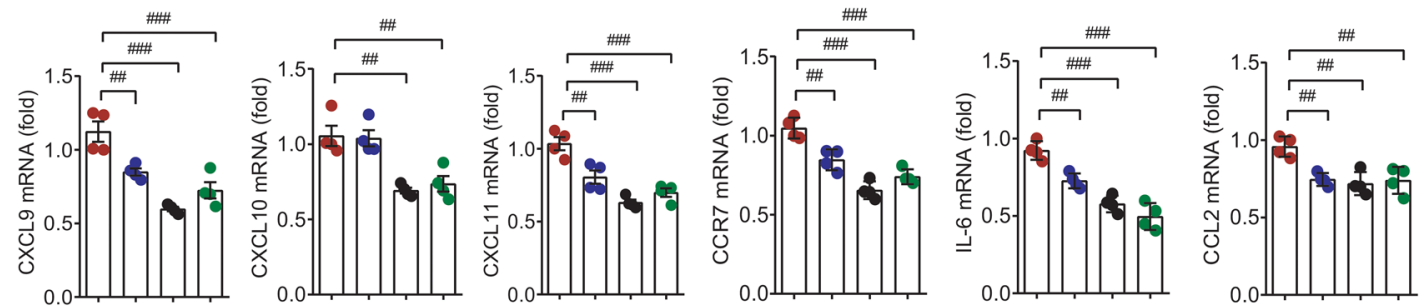

D

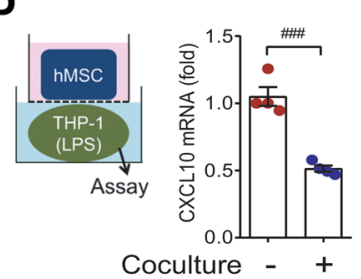

F
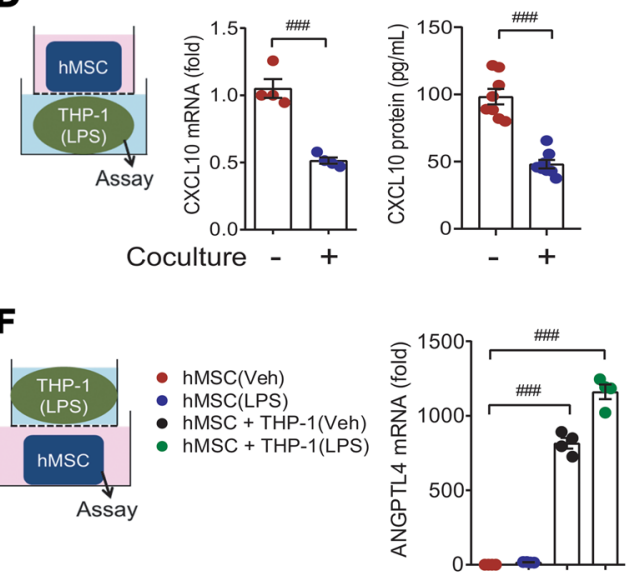

I
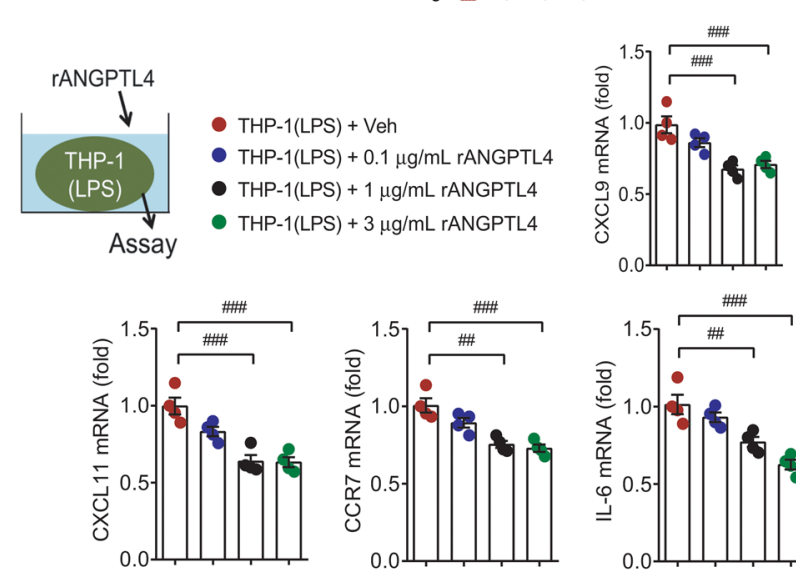

E

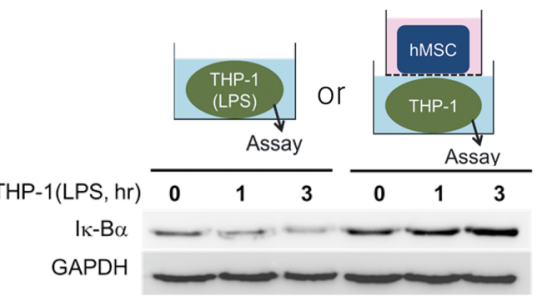

- THP-1(Veh)

- THP-1(LPS) $1 \mathrm{~h}$

- THP-1(LPS) $3 \mathrm{~h}$

O THP-1(Veh) + hMSC

O THP-1(LPS) $1 \mathrm{~h}+\mathrm{hMSC}$

O THP-1(LPS) $3 \mathrm{~h}+\mathrm{hMSC}$

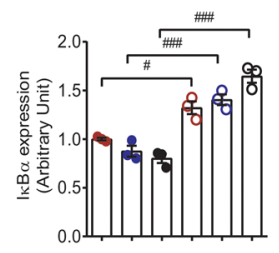

G
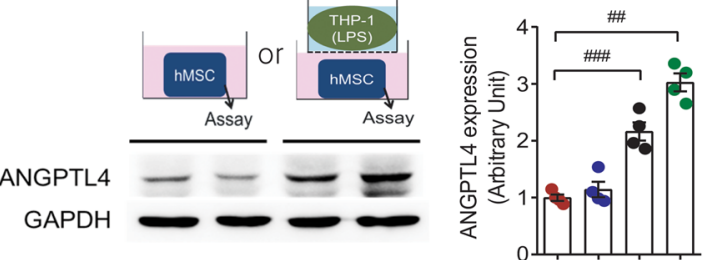

$\mathbf{H}$

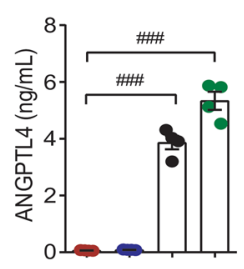


Figure 1. ANGPTL4 derived from MSCs is essential for inhibiting the inflammatory activation of macrophages. (A) Both ANGPTL4 mRNA and protein were notably increased in human mesenchymal stem cells (hMSCs) when cocultured with either unstimulated human primary macrophages (hMFs) or LPS-stimulated hMFs. $n=4$. (B) siRNA control-transfected (siCon-transfected) hMSCs reduced the mRNA levels of inflammatory markers Cxc/9, Cxc/10, Cxc/11, Ccr7, II6, and CcI2 in LPS-stimulated hMFs after coculture for 24 hours, while siRNA ANGPTL4-transfected (siANGPTL4-transfected) hMSCs did not. $n=4$. (C) Inflammatory markers in LPS-stimulated hMFs were significantly reduced by treatment with recombinant ANGPTL4 protein. $n=4$. (D) Differentiated THP-1 macrophages showed significant reductions in proinflammatory CXCL10 mRNA $(n=4)$ and protein induction ( $n=8)$ by LPS stimulation by coculture with hMSCs. (E) LPS-induced degradation of IאB $\alpha$ protein in THP-1 macrophages was blunted by coculture with hMSCs. Western blots are representative of $n>3$ repeats. Intensity quantification is representative of mean \pm SEM. $(\mathbf{F}-\mathbf{H})$ ANGPTL4 mRNA $(n=4)$, cellular protein $(n=4)$, and released protein $(n=4)$ were assayed in hMSCs with or without coculture with LPS-stimulated THP-1 macrophages. (I) ANGPTL4 protein-treated THP-1 macrophages showed significant reductions in inflammatory genes in a dose-dependent manner. $n=4$. Data are represented as mean $\pm S E M$. $\# P<0.05$; $\# \#<0.01 ; \# \# P<0.001$ (by Student's $t$ test or 1-way ANOVA with Bonferroni's multiple-comparisons test).

Next, we sought to determine how hMSCs acted on macrophages to exert their antiinflammatory activity. First, to determine the hMSC-released exosomes, we pretreated the hMSCs with GW4869, a well-known inhibitor of exosome release, to block exosome production, but found no differences in the inhibition of proinflammatory CXCL10 mRNA in THP-1 macrophages (Figure 3A). Then, we pretreated macrophages with cytochalasin $\mathrm{D}$, which is known to inhibit exosome uptake, before coculture with hMSCs, and found no difference in CXCL10 inhibition in macrophages regardless of cytochalasin D pretreatment (Figure 3B). Second, because ANGPTL4 has been reported to bind integrin $\beta_{1}$ or integrin $\alpha_{v} \beta_{3}(5$, 11 ), we neutralized these binding sites on macrophages by antibodies before coculture. Blockade of integrin $\beta_{1}$ and integrin $\alpha_{\mathrm{y}} \beta_{3}$ impaired the inhibition of CXCL10 in THP-1 macrophages by hMSCs (Figure 3C). These results indicated that ANGPTL4 released from MSCs may act on macrophages through integrin subtypes rather than exosomes.

ANGPTL4 governs the profile of inflammatory-soluble mediators in MSCs. Further analysis of the released and intracellular proteins associated with MSCs was performed by antibody array. After coculture with LPS-stimulated THP-1 macrophages, hMSC coculture medium (CCM) and hMSC lysate were collected from both control siRNA-transfected (Figure 4A) and ANGPTL4 siRNA-transfected hMSCs (Figure 4B). CXCL11 and CSF2 were reduced, whereas IL-8 was increased in CCM from ANGPTL4-knockdown hMSCs. In addition, CSF2 and IL-1 $\beta$ were reduced, whereas MIP- $1 \alpha$ and MIP- $1 \beta$ were increased, in ANGPTL4-knockdown hMSCs. To further support these results, we performed real-time PCR, and these factors were shown to change consistently with the antibody array (Figure 4C).

ANGPTL4 in MSCS supports the antiinflammatory effect in a mouse peritonitis model. To functionally show the critical role of ANGPTL4 in MSCs to inhibit pathological inflammation, we collected CCM from wild-type or ANGPTL4-knockout mMSCs cocultured with BMDMs. We induced peritonitis in mice with thioglycollate injection. On day 3, CCM was injected into the peritoneum, and 20 hours later, mice were stimulated by intraperitoneal injection with LPS for 4 hours (Figure 5A). Intriguingly, circulating ANGPTL4 was substantially increased, while IL- 6 was reduced in the wild-type mMSC-CCM group but not in the knockout mMSC-CCM group (Figure 5B). On the other hand, levels of ANGPTL4 and IL-6 were not different in peritoneal lavage in all groups (Figure 5C). We next analyzed inflammation-related mediators of peritoneal macrophages. In a series of real-time PCR experiments, the peritoneal macrophages isolated from the wild-type mMSC-CCM group showed higher expression of antiinflammatory Arg1, CD206, and IL-10 and lower levels of proinflammatory iNOS, IL-6, IL-1 $\beta$, and CD80 compared with the knockout mMSC-CCM group (Figure 5D).

Additionally, we supported the essential role of ANGPTL4 in antiinflammatory activity of MSCs by using CCM collected from hMSCs with ANGPTL4 knockdown and THP-1 macrophages. The inhibitory effect of hMSC(siANGPTL4)-CCM on the induction of CXCL10 and CXCL11 was not observed in LPS-stimulated THP-1 macrophages (Supplemental Figure 5A). In a peritonitis mouse model, circulating ANGPTL4 was increased, while IL-6 was reduced in the hMSC(siCon)-CCM group but not in the hMSC(siANGPTL4)-CCM group. However, peritoneal lavage did not show any differences in the levels of ANGPTL4 and IL-6 in either group (Supplemental Figure 5B). The antiinflammatory protein CD206 and antiinflammatory genes ( $\mathrm{Arg} 1, \mathrm{Cd} 206$, and Il10) were highly expressed in the peritoneal macrophages isolated from the hMSC(siCon)-CCM group compared with the hMSC(siANGPTL4)-CCM group (Supplemental Figure 5C). The representative antiinflammatory marker CD206 was upregulated in the peritoneal macrophages isolated from the $\mathrm{hMSC}$ (siCon)-CCM group but not in the hMSC(siANGPTL4)-CCM group (Supplemental Figure 5D). Inflammation-related cytokines were quantified 
A

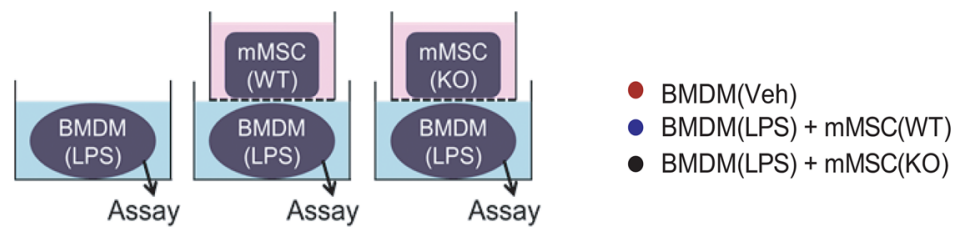

Anti-inflammatory Markers (24 h)
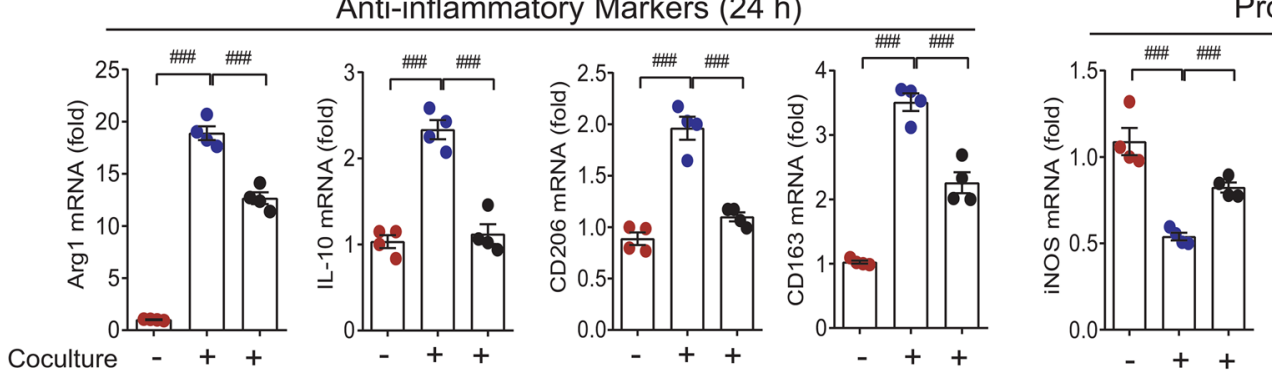

Pro-inflammatory Markers (24 h)

Anti-inflammatory Markers (48 h)
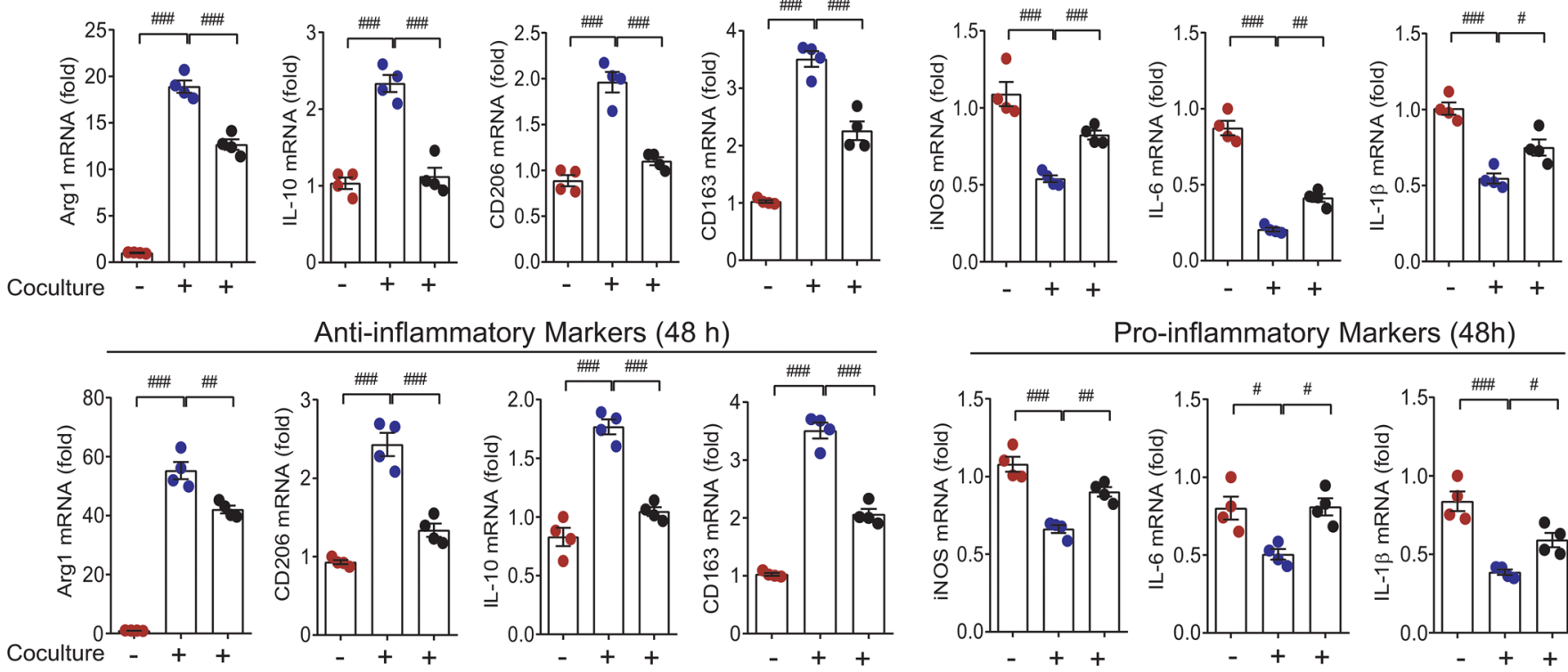

Pro-inflammatory Markers (48h)

\section{B}
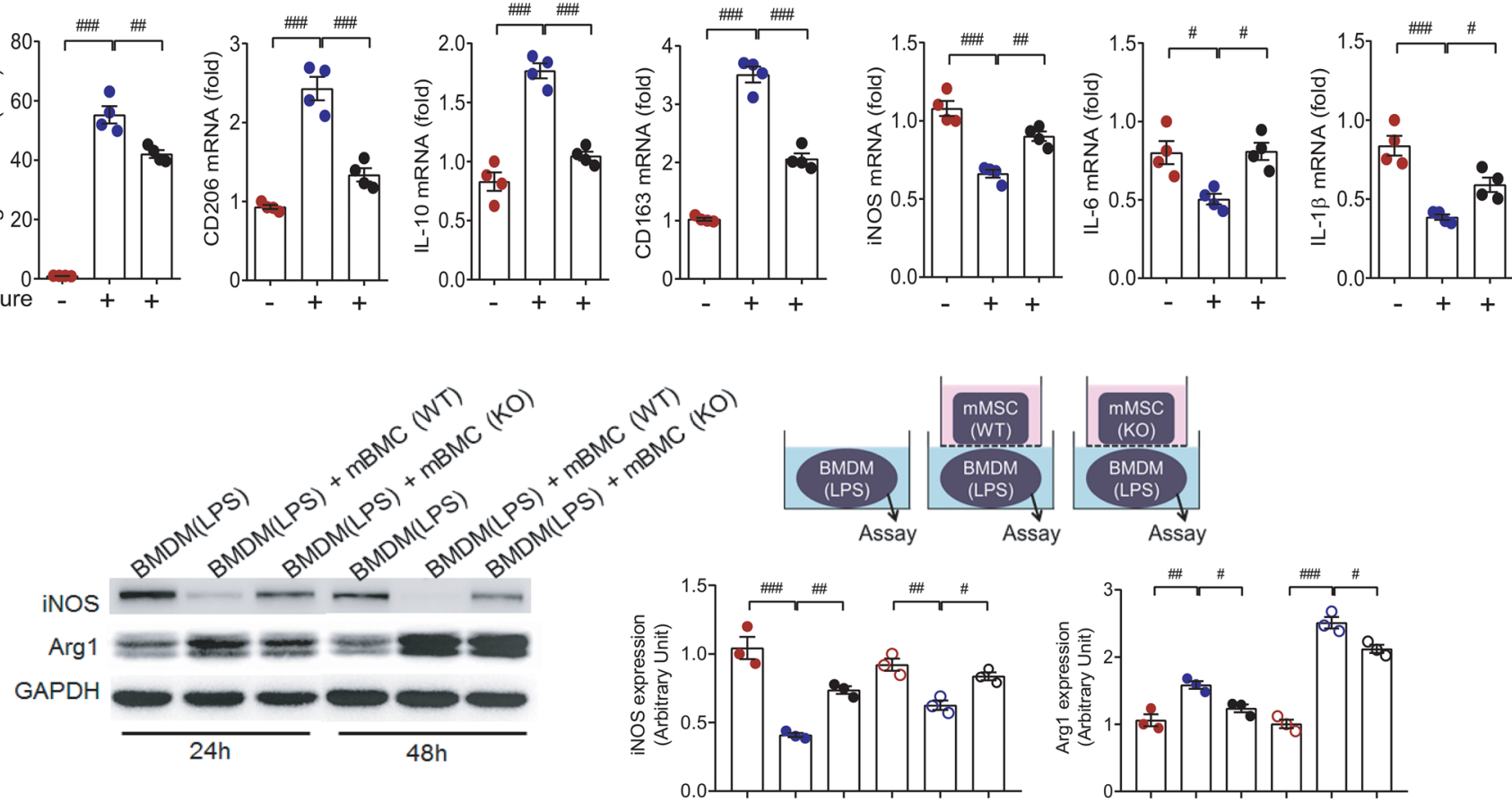

Figure 2. Deficiency of ANGPTL4 in MSCs fails to suppress inflammatory activation of macrophages. (A) Mouse bone marrow-derived MSCs (mMSCs) were isolated from wild-type and ANGPTL4-knockout mice. In coculture settings, inflammation-related markers were assessed in LPS-stimulated mouse bone marrow-derived macrophages (BMDMs) with or without coculture with mMSCs at 24 hours and 48 hours. Wild-type mMSCs significantly suppressed proinflammatory markers, while knockout mMSCs did not. Antiinflammatory markers in macrophages were remarkably induced only by wild-type mMSCs but not by knockout mMSCs. $n=4$. (B) In the same setting as A, protein levels of iNOS and Arg1 were measured by Western blot. Intensity quantification is representative of mean \pm SEM. $n=3$. Data are represented as mean \pm SEM. ${ }^{\#} P<0.05$; ${ }^{\# \#} P<0.01 ; \# \# P<0.001$ (by Student's $t$ test or 1 -way ANOVA with Bonferroni's multiple-comparisons test).

in mouse plasma by using multiplex ELISA. IL-1 $\alpha$ and IL-6 were lower in the hMSC(siCon)-CCM group than in the MSC(siANGPTL4)-CCM group (Supplemental Figure 5E).

ANGPTL4 supports the antiinflammatory effect in a mouse peritonitis model. To confirm that ANGPTL4 was responsible for the antiinflammatory activity of MSC CCM, we injected Veh or recombinant ANGPTL4 protein ( $50 \mathrm{ng}$ or $200 \mathrm{ng}$ ) into mice with peritonitis (Figure 6A). Plasma IL-6 was reduced in both the 50-ng ANGPTL4 group and the 200-ng ANGPTL4 group, whereas circulating ANGPTL4 did not differ (Figure 6B). A series of real-time PCRs of peritoneal macrophages showed that the 200-ng ANGPTL4 group had significantly increased expression of the antiinflammatory Arg1, CD206, and IL-10 and decreased proinflammatory iNOS, IL-6, and IL-1 $\beta$. On the other hand, 50-ng ANGPTL4 treatment showed a partial 
A
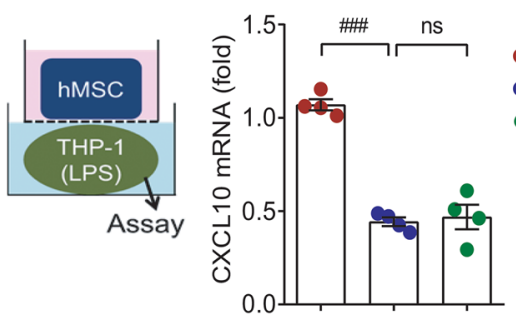

THP-1(LPS)

- THP-1(LPS) + hMSC(Veh)

THP-1(LPS) + hMSC(GW4869)
B

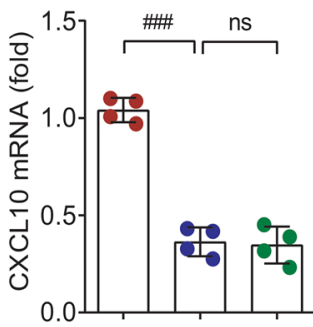

- THP-1(LPS)

- THP-1(LPS) + hMSC

- THP-1(LPS, Cytochalasin D) + hMSC
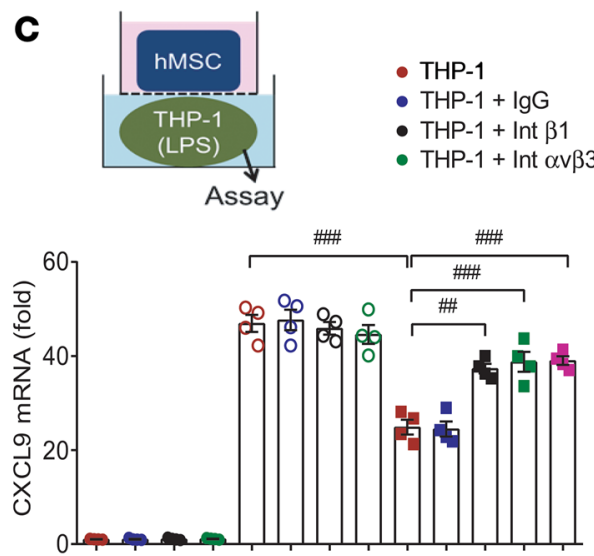

- THP-1

- THP-1 + lgG

- THP-1 + Int $\beta 1$

- THP-1 + Int $\alpha \mathrm{v} \beta 3$

- THP-1(LPS) + IgG

o $\mathrm{THP}-1(\mathrm{LPS})+\operatorname{Int} \beta 1$

o THP-1(LPS) + Int $\alpha v \beta 3$
- THP-1(LPS) + hMSC

- THP-1(LPS) + lgG + hMSC

- THP-1(LPS) + Int $\beta 1+$ hMSC

- THP-1(LPS) + Int $\alpha v \beta 3+$ hMSC

- THP-1(LPS) + Int $\beta 1+$ Int $\alpha v \beta 3+$ hMSC
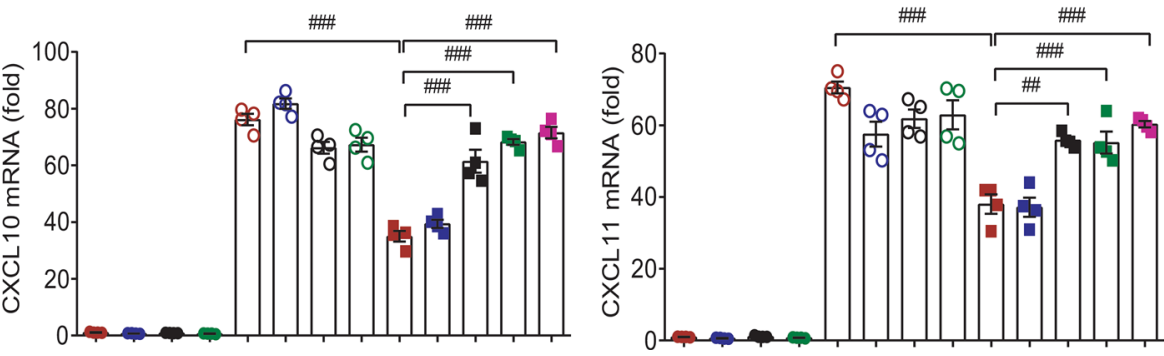

Figure 3. Antiinflammatory activity of MSCs is mediated by integrin subtypes on macrophages, not by exosomes. (A) hMSCs were pretreated with CW4869, a blocker of exosome production, before coculture with THP-1 macrophages. Cene expression of CXCL10 of macrophages was assessed after coculture with vehicle or GW4869-treated hMSCs. $n=4$. (B) THP-1 macrophages were pretreated with cytochalasin D, an inhibitor of exosome uptake, before coculture with hMSCs. $n=4$. (C) Coculture with hMSCs does not suppress inflammatory activation of macrophages pretreated with neutralizing antibody against integrin $\beta_{1}$ or integrin $\alpha_{v} \beta_{3}$. Thus, integrin subtypes on THP-1 macrophages may be involved in the antiinflammatory action of ANGPTL4 released from hMSCs. $n=4$. Data are represented as mean $\pm S E M$. ${ }^{\# \# ~} P<0.01$; ${ }^{\# \#} P<0.001$ (by Student's $t$ test or 1 -way ANOVA with Bonferroni's multiple-comparisons test).

inhibitory effect on inflammation (Figure 6C). In plasma cytokine analyses, significant reductions of IL-6, IL-1 $\alpha$, IL-1 $\beta$, and IL-10 upregulation were observed only in the 200-ng ANGPTL4 group and not in the 50-ng ANGPTL4 group (Figure 6D). CD206-expressing peritoneal macrophages were more abundant in the group treated with $200 \mathrm{ng}$ ANGPTL4 than in the Veh-treated group (Figure 6E). In addition to peritoneal macrophages, we assessed the antiinflammatory effect of 200-ng ANGPTL4 treatment in BMDMs isolated from mice with peritonitis. As in the peritoneal macrophages, antiinflammatory genes were increased, whereas proinflammatory markers were downregulated in the LPS-stimulated BMDMs from the ANGPTL4 group (Figure 6F). From these results, we suggest that treatment with 200 ng ANGPTL4 protein was comparable to MSC CCM in terms of antiinflammatory activity.

ANGPTL4 in MSCs eases cardiac inflammation and improves cardiac function. In the peritonitis model, we noticed that ANGPTL4 treatment exerted its antiinflammatory effect against pathologically activated macrophages. Next, to address the relevance of our observations for advanced cardiac inflammation, we used an MI model as summarized in Figure 7A. CCM was injected into the mouse intraperitoneally, followed by MI induction. At 2 days, plasma and heart tissues were collected. Inflammatory IL- 6 and IL-1 $\beta$ in the plasma were reduced by the injection of wild-type mMSC CCM but not by knockout mMSC CCM (Figure 7B). In heart tissues, antiinflammatory Arg1, CD206, and IL-10 were increased, while proinflammatory iNOS, IL-6, IL-1 $\beta$, and MCP-1 were reduced in the wild-type mMSC-CCM group but not in the knockout mMSC-CCM group (Figure 7C). In Western blots, the antiinflammatory markers Arg1 and CD206 were not upregulated, and the proinflammatory markers iNOS and MCP-1 were not downregulated in the knockout mMSC-CCM group (Figure 7D). To lend further support to these findings, hMSC(siANGPTL4) CCM was applied to the MI model (Supplemental Figure 6A). In the hMSC(siANGPTL4)-CCM group, no antiinflammatory effects of CCM were shown in the circulating levels of IL- 6 and IL- $1 \beta$ or the expression patterns of antiinflammatory markers and proinflammatory markers (Supplemental Figure 6, B-D). 
A

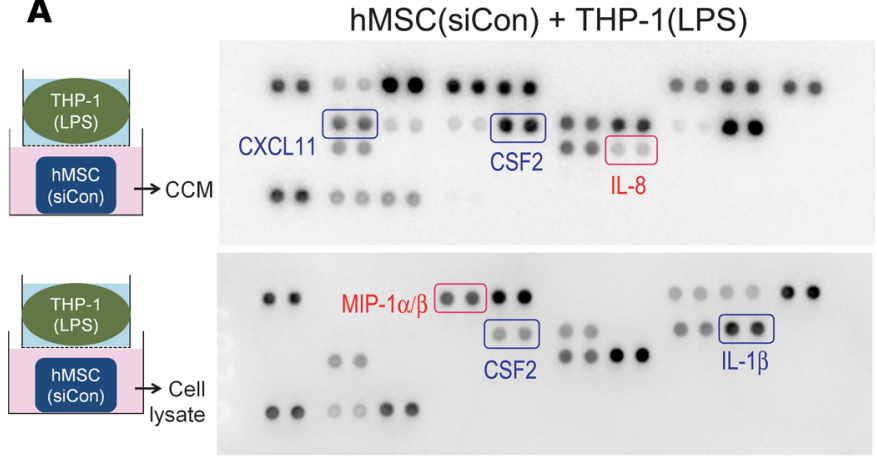

B

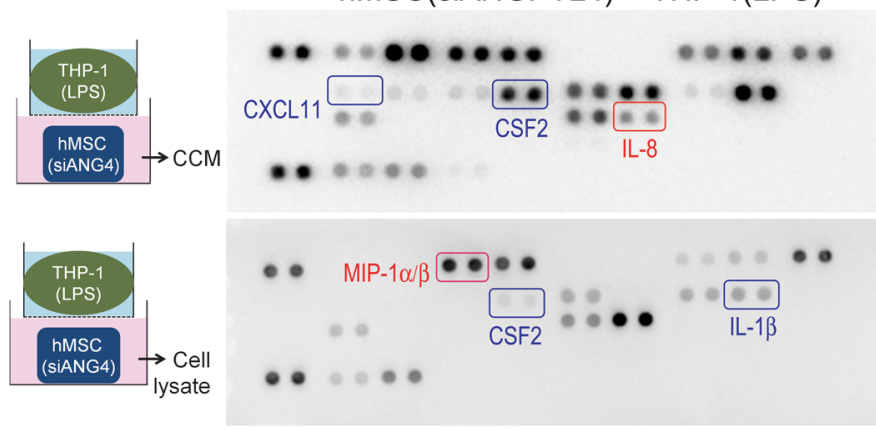

C

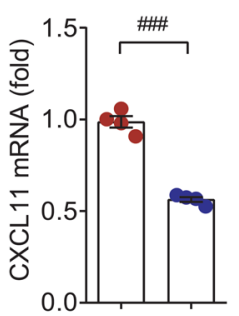

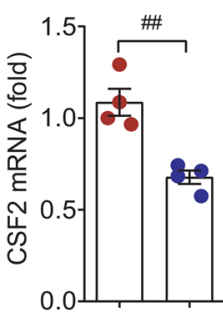

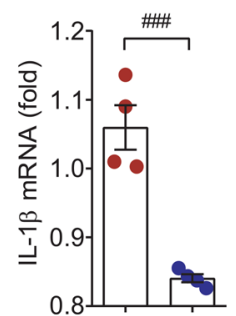

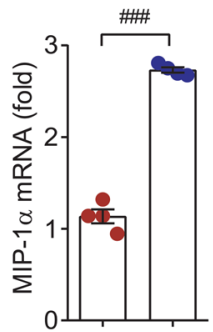
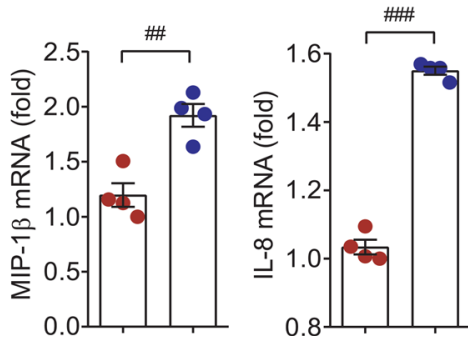

Figure 4. Soluble factors released from MSCs display antiinflammatory potential. Antibody array was used to analyze soluble factors of hMSCs. (A and B) hMSCs were transfected with control siRNA or ANGPTL4 siRNA before coculture with LPS-stimulated THP-1 macrophages. hMSC coculture medium (CCM, upper membranes) and hMSC lysate (lower membranes) were analyzed by antibody array to compare soluble factor expression. $n=3$. (C) $\mathrm{mRNA}$ levels of the differentially expressed proteins detected by antibody array were measured by real-time PCR. $n=4$. Data are represented as mean \pm SEM. ${ }^{\#} P<0.05 ;{ }^{\# \#} P<0.01 ;{ }^{\# \# P}<0.001$ (by Student's $t$ test).

ANGPTL4 eases cardiac inflammation and improves cardiac function. To evaluate the functional role of ANGPTL4 in MI, ANGPTL4 protein was injected every other day into MI mice. At 2 days, plasma and heart tissues were collected (Figure 8A). In heart tissues, antiinflammatory markers were increased, while proinflammatory markers were reduced, in the ANGPTL4 group (Figure 8, B and C). Circulating IL-6 and IL-1 $\beta$ were reduced by the injection of ANGPTL4 (Figure 8D). To further analyze the effect of ANGPTL4 on the phenotype of cardiac macrophages, mice were subjected to MI for 3 days or 2 weeks (Figure 8E). At day 3 after MI, inflammatory cardiac macrophages, which were identified by sorting with $C D 45^{\text {hi }} \mathrm{CD}$ $11 \mathrm{~b}^{+} \mathrm{F} 4 / 80^{+} \mathrm{CD} 80^{\text {hi }}$ cells more abundant in the Veh group than in the ANGPTL4 group (Figure 8F). Moreover, mRNA levels of antiinflammatory Arg1 and Cd206 were higher in the cardiac macrophages isolated from the MI mice injected with ANGPTL4 protein (Figure 8G). These results led us to analyze the therapeutic effect of ANGPTL4 protein in the post-MI condition. At 2 weeks after MI, heart tissues were collected, and cardiac function was assessed by echocardiography and immunohistochemistry (Figure $8 \mathrm{H}$ ). The gross sections of the heart tissues showed better preserved cardiac structure in the group treated with ANGPTL4 than in the Veh-treated group (Supplemental Figure 7A), and cardiac fibrosis was attenuated in the group treated with ANGPTL4 (Supplemental Figure 7B). To compare macrophage infiltration, CD68 ${ }^{+}$macrophages were visualized. We found that the distribution of macrophages was smaller in the group treated with ANGPTL4 (Supplemental Figure 7C). The distribution of the antiinflammatory marker CD206 was higher in the group treated with $200 \mathrm{ng}$ ANGPTL4 than in the Veh group (Supplemental Figure 7D). Additionally, when we assessed the distribution of $\mathrm{vWF}^{+}$cells, we found that angiogenesis in the infarcted zone was better in the group treated with ANGPTL4 than in the Veh-treated group (Supplemental Figure 7E).

To determine the relevance of ANGPTL4 to cardiac pathology, we measured cardiac function. Echocardiographic indexes indicated that cardiac remodeling and function were improved in the group treated with 200 ng ANGPTL4 compared with Veh (Figure 8I). Next, we analyzed the phenotype of cardiac macrophages by double staining with $\mathrm{CD} 68$ and $\mathrm{CD} 206$ and found that CD206-expressing macrophages were more abundant in the ANGPTL4 group than in the Veh group (Figure 8J).

To further support the cardioprotective effect of ANGPTL4, we examined the effect of ANGPTL4 on cardiomyocytes and cardiac fibroblasts in addition to macrophages. Neonatal cardiomyocytes and cardiac fibroblasts 
A
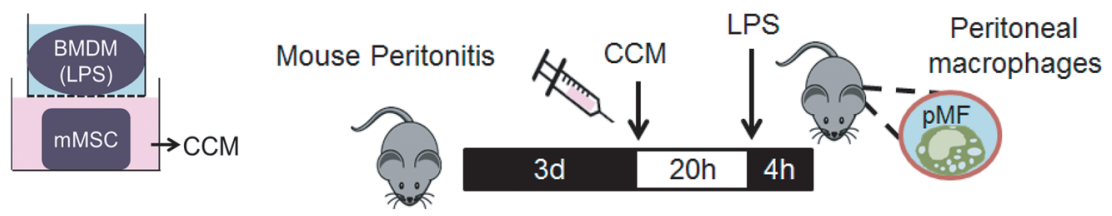

B

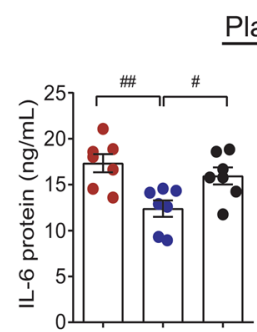

Plasma

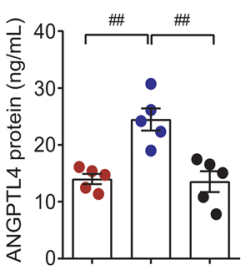

C

- Veh

- mMSC(WT)-CCM

- mMSC(KO)-CCM

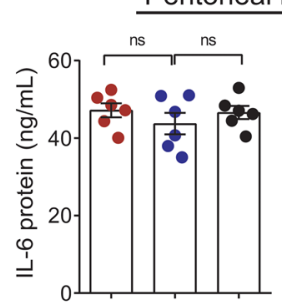

- Veh

- mMSC(WT)-CCM

- $\mathrm{mMSC}(\mathrm{KO})-\mathrm{CCM}$

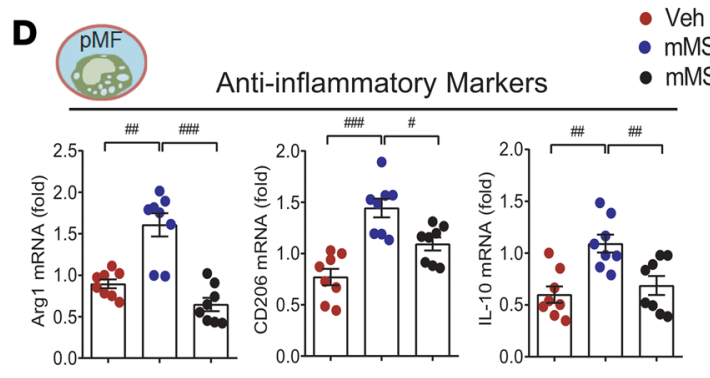

- Veh

mMSC(WT)-CCM

$\mathrm{mMSC}(\mathrm{KO})-\mathrm{CCM}$

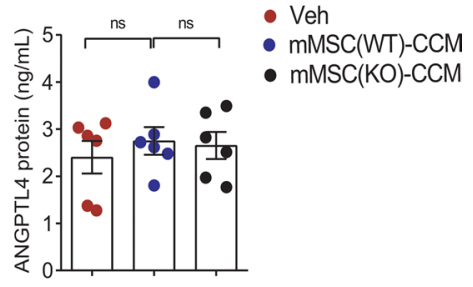

Pro-inflammatory Markers

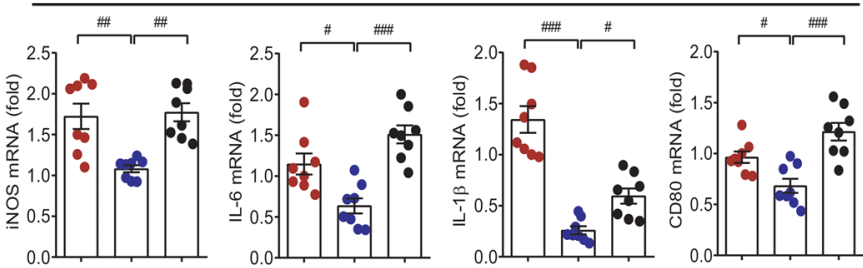

Figure 5. ANGPTL4 is essential for MSCs to suppress peritoneal macrophage activation. (A) Experimental procedure with mouse peritonitis. Peritonitis was induced by thioglycollate injection for 3 days. CCM collected from wild-type mMSCs or knockout mMSCs was injected into the peritoneum followed by LPS stimulation for 4 hours. Peritoneal macrophages were isolated for further analyses. Circulating ANGPTL4 and IL-6 in plasma (B) and peritoneal lavage (C) were quantified. (D) Inflammation-related genes were assessed in peritoneal macrophages collected from mice with peritonitis $(n=8)$. Mice were injected with vehicle (Veh) or CCM i.p. on days 0 and $2 . n=5$ for circulating ANGPTL4; $n=7$ for circulating IL-6; $n=$

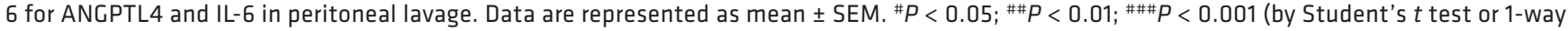
ANOVA with Bonferroni's multiple-comparisons test).

were stimulated with angiotensin II (Ang II), a major mediator of cardiac damage (12). In cardiomyocytes, Ang II-induced upregulation of apoptotic protein BAX was significantly suppressed, while antiapoptotic BCL2 was highly induced by ANGPTL4 treatment (Supplemental Figure 7F). Then the effect of ANGPTL4 on Ang IIinduced profibrotic mediators was assessed. Connective tissue growth factor (CTGF) was substantially inhibited in cardiomyocytes (Supplemental Figure 7G), and collagen (Colla1) and CTGF in cardiac fibroblasts were also remarkably inhibited by ANGPTL4 treatment (Supplemental Figure 7H). Thus, in the infarcted heart, ANGPTL4 may exert its therapeutic effect on macrophages, cardiomyocytes, and cardiac fibroblasts.

Identification of retinoic acid receptor-related orphan receptor $\alpha$ as a transcriptional inducer of ANGPTL4. Next, we explored transcription factor binding sites of the ANGPTL4 promoter to address how ANGPTL4 is induced in MSCs and found that retinoic acid receptor-related orphan receptor $\alpha(\mathrm{ROR} \alpha)$ was a candidate transcription factor with a binding site on the ANGPTL4 promoter. ROR $\alpha$ was induced when hMSCs were cocultured with THP-1 macrophages (Supplemental Table 1 and Figure 9A). To examine whether ROR $\alpha$ is required for ANGPTL4 transcriptional activity, we knocked down ROR $\alpha$ and found that ANGPTL4 upregulation was blunted in hMSCs with ROR $\alpha$ knockdown transcriptionally and translationally (Figure 9 , B and C). Alternatively, ROR $\alpha$-overexpressed hMSCs showed significant upregulation of ANGPTL4 (Supplemental Figure 8A). Besides, hMSCs with ROR $\alpha$ knockdown failed to suppress CXCL10 and CXCL11 in LPS-stimulated macrophages (Figure 9D).

Then, to study whether inflammatory cytokines released from infiltrated macrophages may induce ANGPTL4 in MSCs $(13,14)$, we treated hMSCs with IL-1 $\beta$ or TNF- $\alpha$. Both ROR $\alpha$ and ANGPTL4 were upregulated in hMSCs treated with IL-1 $\beta$ or TNF- $\alpha$ (Figure 9E), and these results suggested that inflammatory mediators derived from macrophages contributed to ANGPTL4 upregulation through ROR $\alpha$ induction in MSCs. Induction of ROR $\alpha$ and ANGPTL4 by treatment with IL-1 $\beta$ or TNF- $\alpha$ was blunted in hMSCs with ROR $\alpha$ knockdown (Figure 9F) 
A

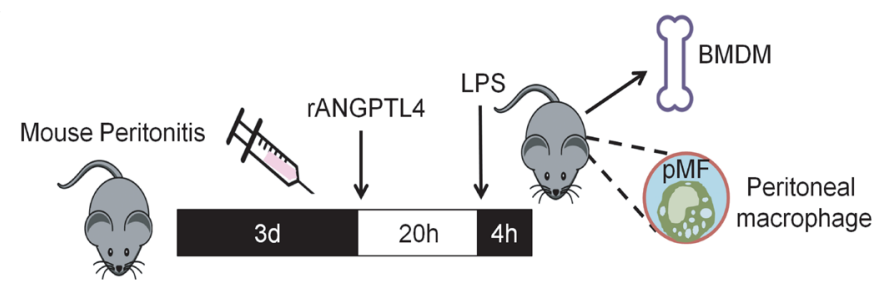

B

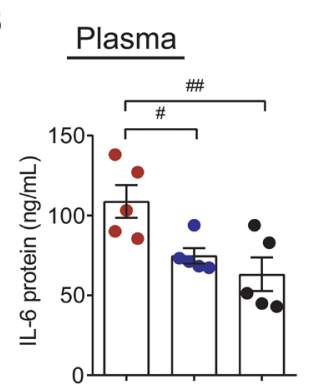

- Veh

- 50 ng rANGPTL4

- 200 ng rANGPTL4

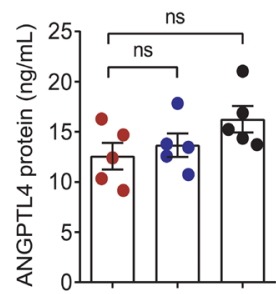

C

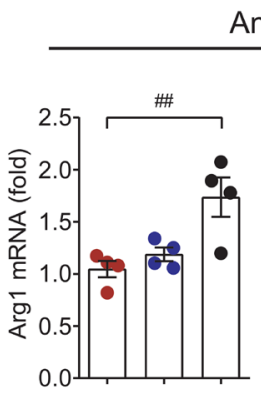

Anti-inflammatory Markers

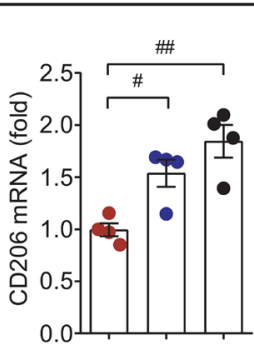

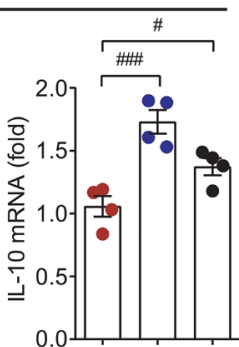

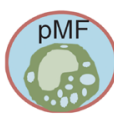

Pro-inflammatory Markers

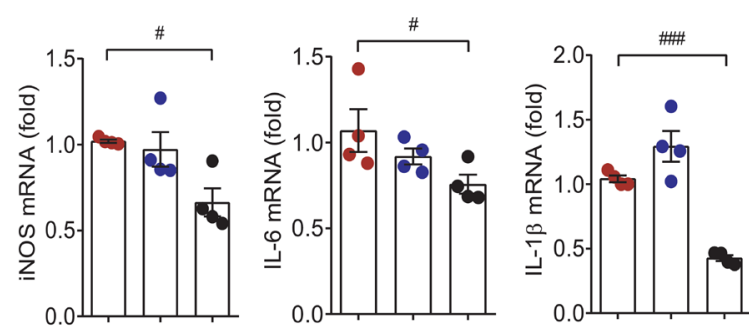

D
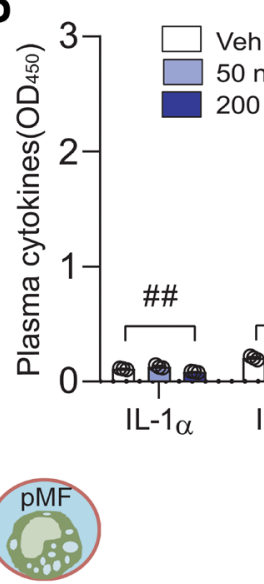

Veh
50 ng rANGPTL4
200 ng rANGPTL4

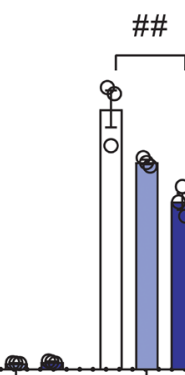

\#\#
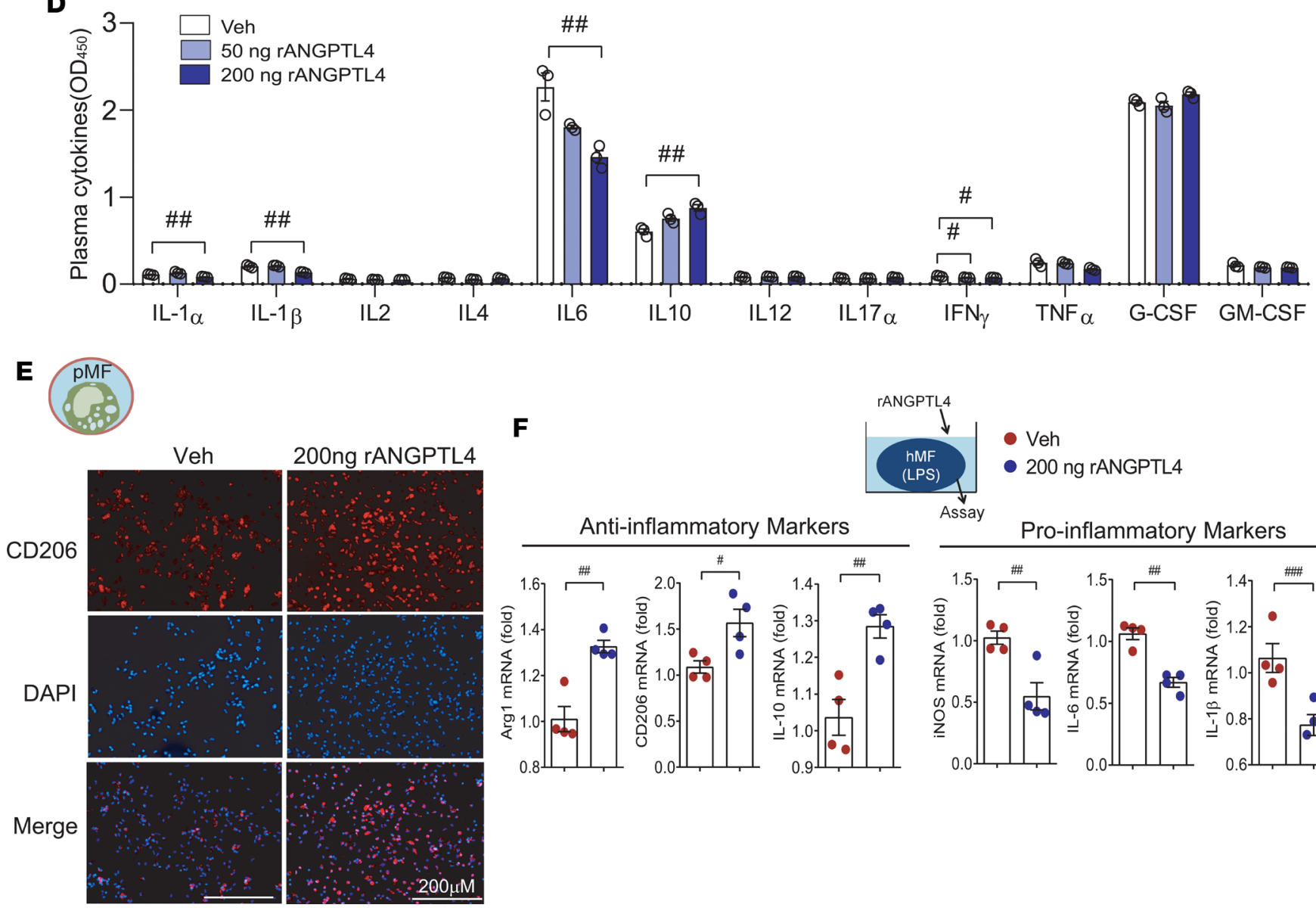

IL4
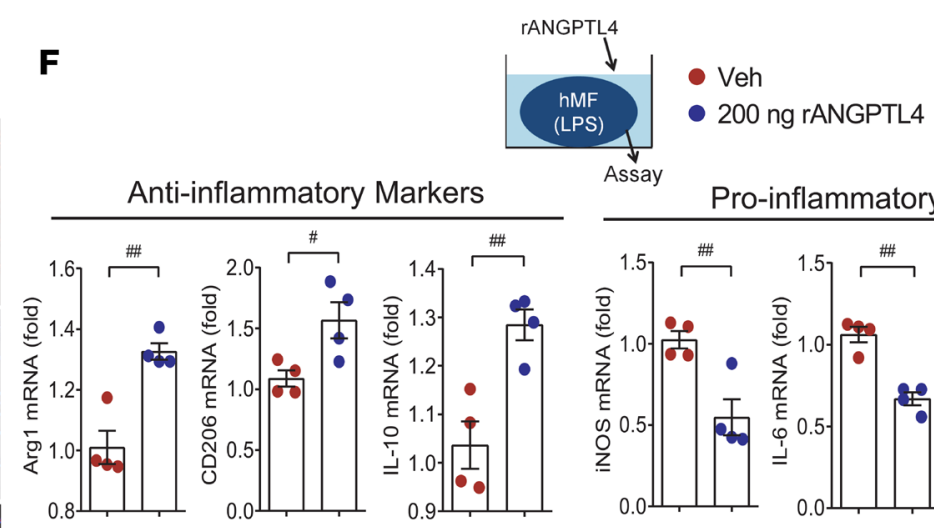

200ng rANGPTL4

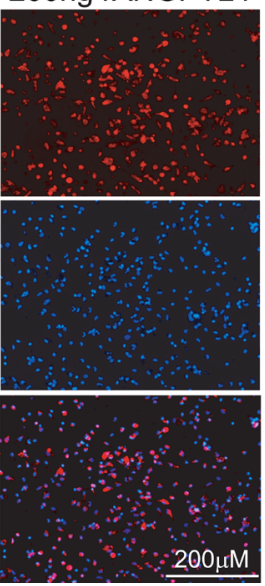

Assay Pro-inflammatory Markers

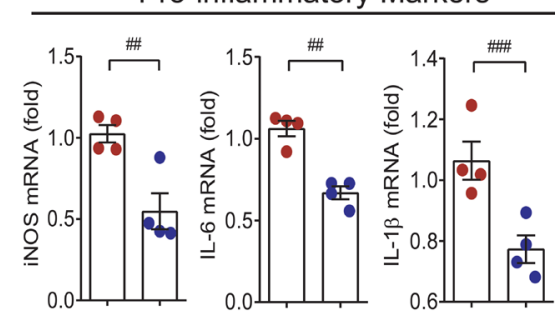

Figure 6. Recombinant ANGPTL4 suppresses the activation of peritoneal macrophages and BMDMs. (A) Veh or recombinant ANGPTL4 protein (50 or 200 ng) was injected to mice with peritonitis. Peritoneal macrophages (pMFs) and BMDMs were used to examine the antiinflammatory effect of ANGPTL4 treatment in a peritonitis mouse model. (B) Circulating IL-6 and ANGPTL4 were measured in the Veh group, 50-ng ANGPTL4 group, and 200-ng ANGPTL4 group. $n=5$ for each group. (C) Inflammation-related genes of peritoneal macrophages were assessed. $n=4$. (D) Inflammatory mediators in the plasma were analyzed by multiplex ELISA. $n=3$. (E) Expression of CD206 was assessed by immunofluorescence staining in peritoneal macrophages. (F) Inflammation-related genes were assessed and the antiinflammatory phenotype was observed in BMDMs isolated from the ANGPTL4 group. $n=4$. Data are represented as mean \pm SEM. ${ }^{\#} P<0.05 ;{ }^{\# \#} P<0.01$; ${ }^{\# \#} P<0.001$ (by Student's $t$ test or 1-way ANOVA with Bonferroni's multiple-comparisons test). 
A

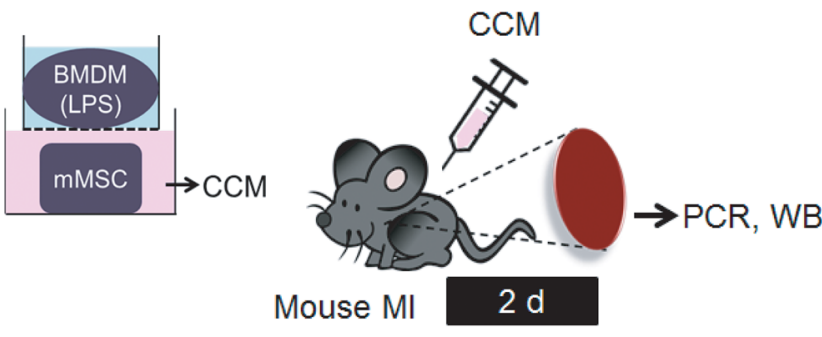

B

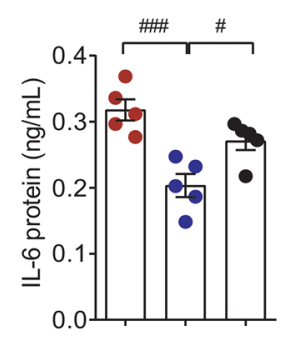

Plasma

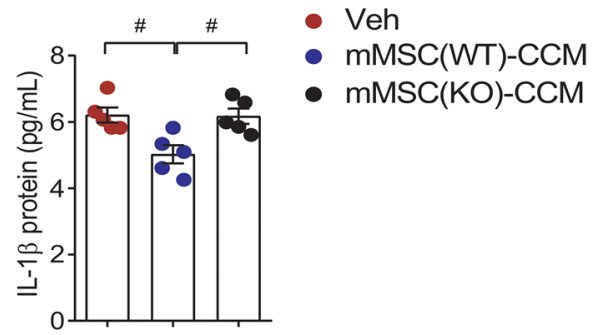

C

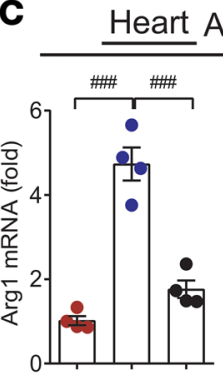

eart Anti-inflammatory Markers

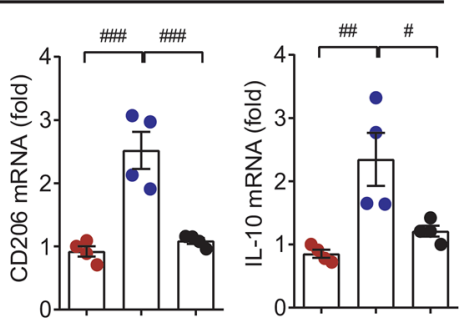

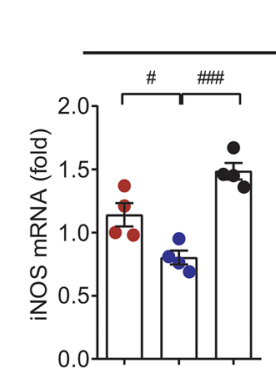

Heart Pro-inflammatory Markers
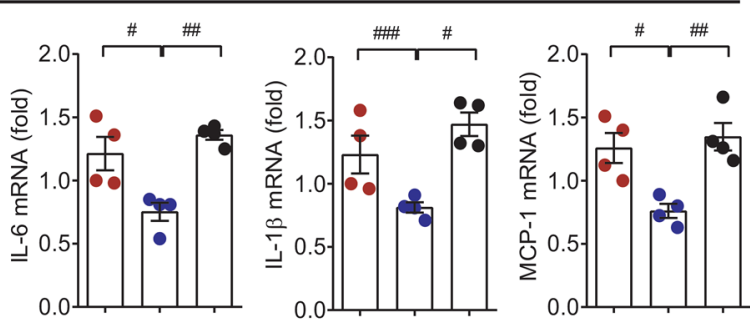

D

Heart
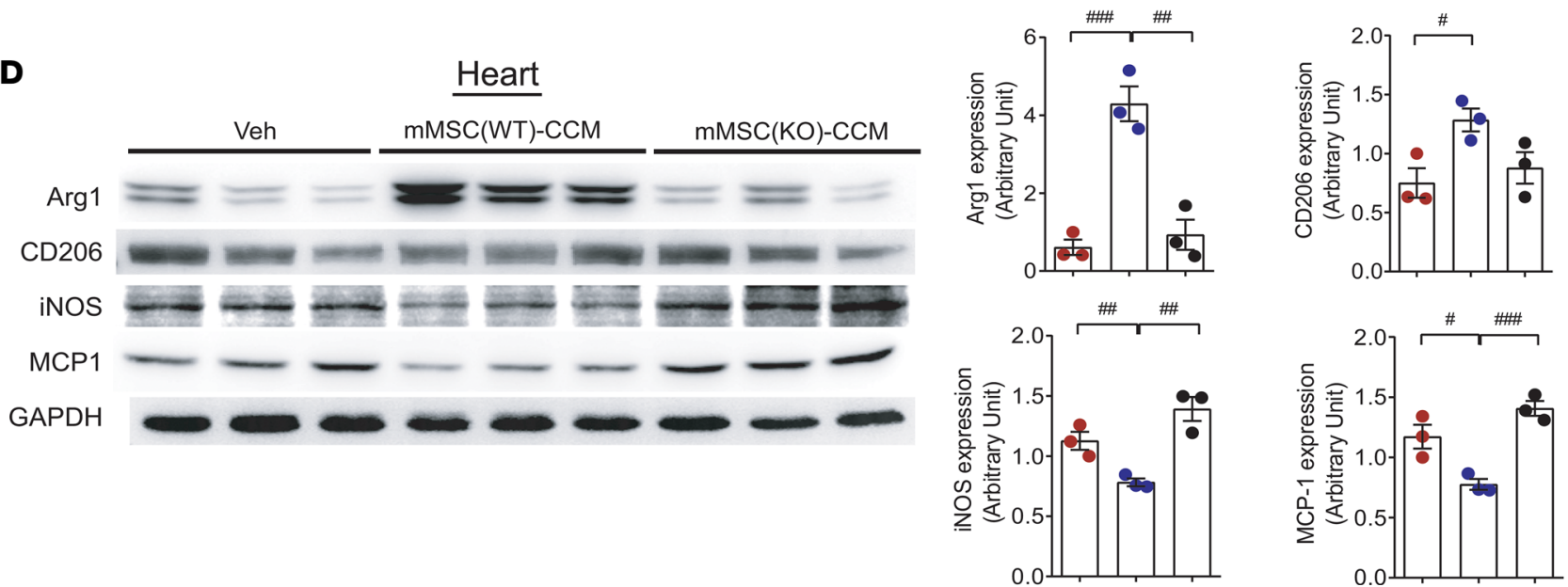

Figure 7. Cardiac inflammation is attenuated by coculture medium in a mouse myocardial infarction model. (A) MI was induced by coronary artery ligation, then CCM from wild-type mouse mMSCs or ANGPTL4-knockout mMSCs was injected i.p. Heart tissues and blood were collected after 2 days for further studies. (B) Circulating IL- 6 and IL-1 $\beta$ were not reduced in mice injected with knockout mMSC CCM. $n=5$ for each group. The expression levels of inflammatory markers in the heart tissue were assessed by real-time PCR $(\mathbf{C}, n=4)$ and Western blot $(\mathbf{D}, n=3)$. Antiinflammatory activity was not observed in the knockout mMSC-CCM group. Data are represented as mean \pm SEM. ${ }^{\#} P<0.05 ;{ }^{\#} P<0.01$; ${ }^{\# \# P} P$. 0.001 (by Student's $t$ test or 1-way ANOVA with Bonferroni's multiple-comparisons test).

Furthermore, pretreatment of hMSCs with SR1001, an inverse agonist of ROR $\alpha$ and ROR $\gamma$, or SR3335, a selective inverse agonist of ROR $\alpha$, failed to upregulate ANGPTL4 as well as ROR $\alpha$ in the coculture with THP-1 macrophages (Figure 9G). Besides, SR1078, an agonist of ROR $\alpha$, substantially increased Rora mRNA in hMSCs along with ANGPTL4 induction (Supplemental Figure 8B). This result showed the association of ROR $\alpha$ and ANGPTL4 in hMSCs in response to inflammatory macrophages. We then evaluated the binding of ROR $\alpha$ on the ANGPTL4 promoter by ChIP assay. Chromatin fragments were immunoprecipitated with a nonspecific IgG as a negative control or with a specific anti-ROR $\alpha$ antibody, and real-time PCR analysis of immunoprecipitated chromatin showed that ROR $\alpha$ bound to the ANGPTL4 promoter (Figure 9H). To find the critical regions in the ANGPTL4 promoter, we established ANGPTL4 promoter-LUC reporter plasmids: full-length promoter and a series of deleted promoters. Deletion mutants were transfected into HEK293T cells, which were stimulated with hMSC CCM. Interestingly, the promoter activity of cells transfected with pGL3-1700 promoter was apparently reduced (about 50\%-60\% reduction) compared with that of cells transfected with 
A $\quad$ Veh

- 50ng rANGPTL4

- 200ng rANGPTL4

B
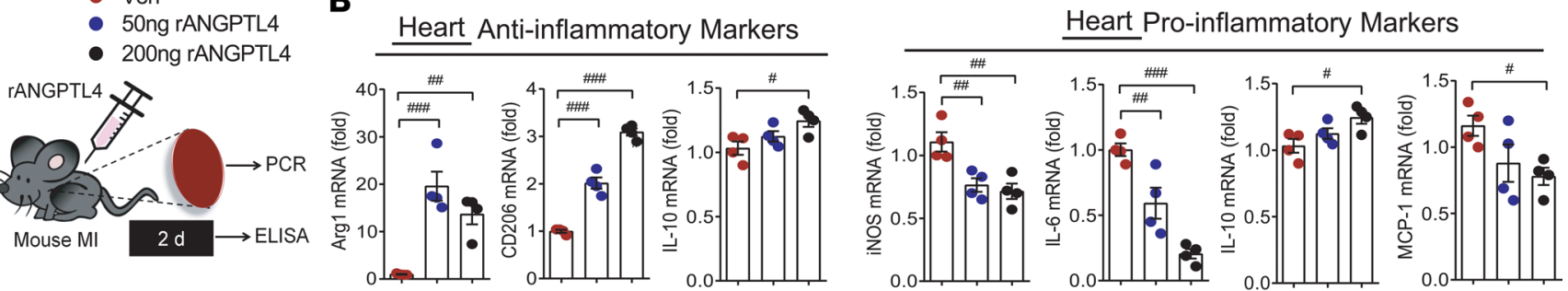

C

Heart
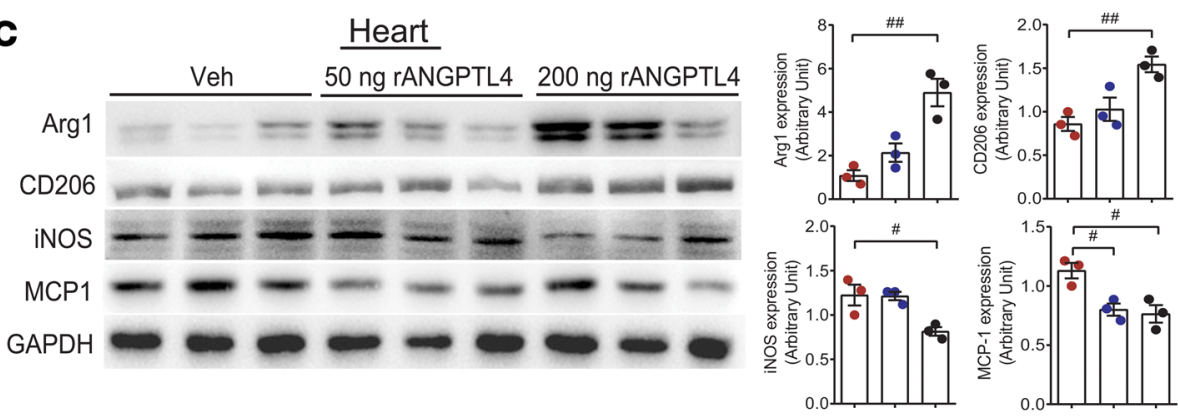

D

$\underline{\text { Plasma }}$

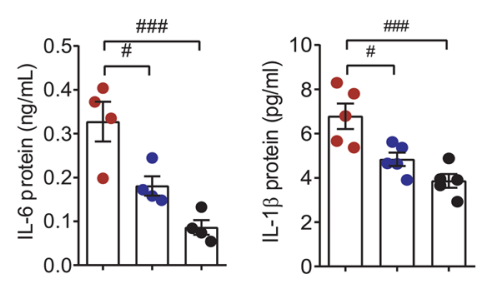

E

F
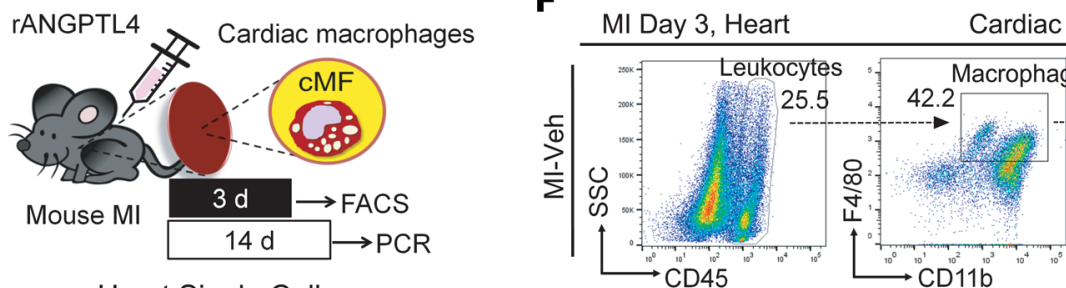

Cardiac $\mathrm{CD} 80^{+}$macrophages

G Heart Single Cells
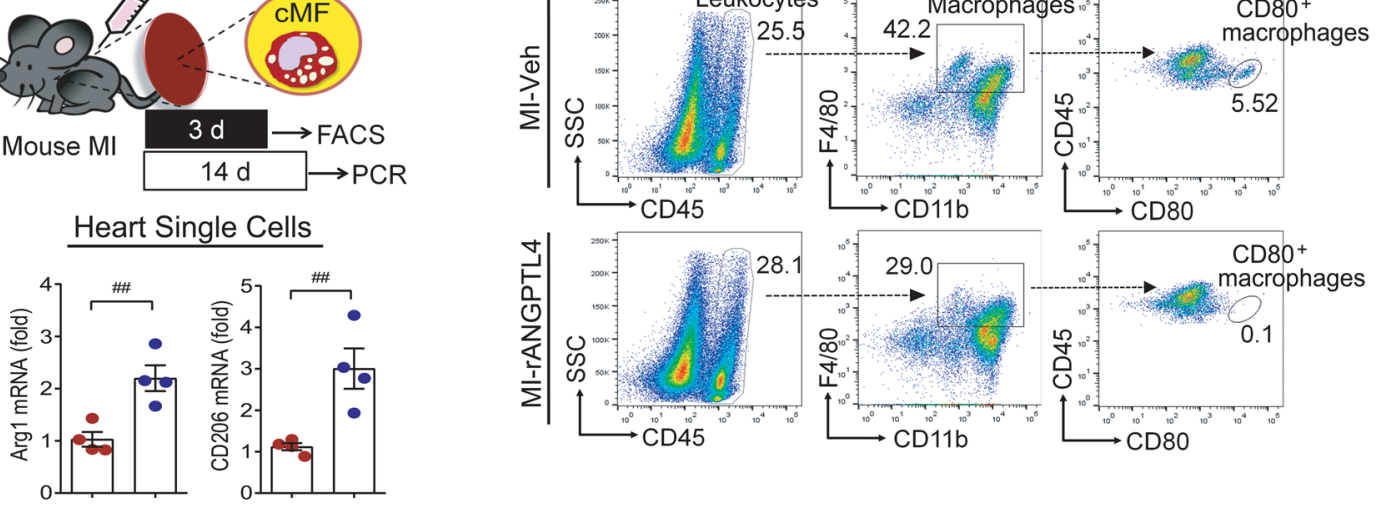

MI-rANGPTL4

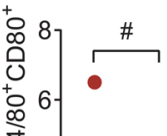
足

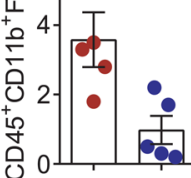

H
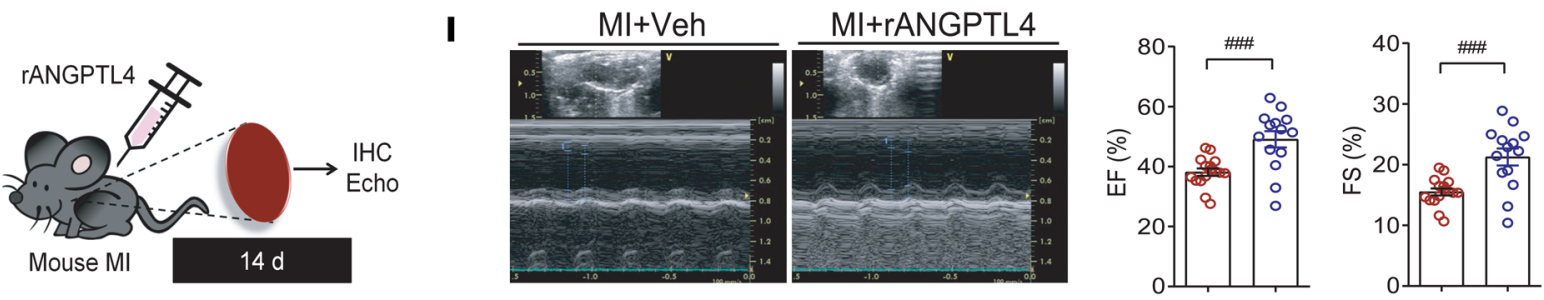

$O \mathrm{Ml}+\mathrm{Veh}$

o $\mathrm{Ml}+$ rANGPTL4
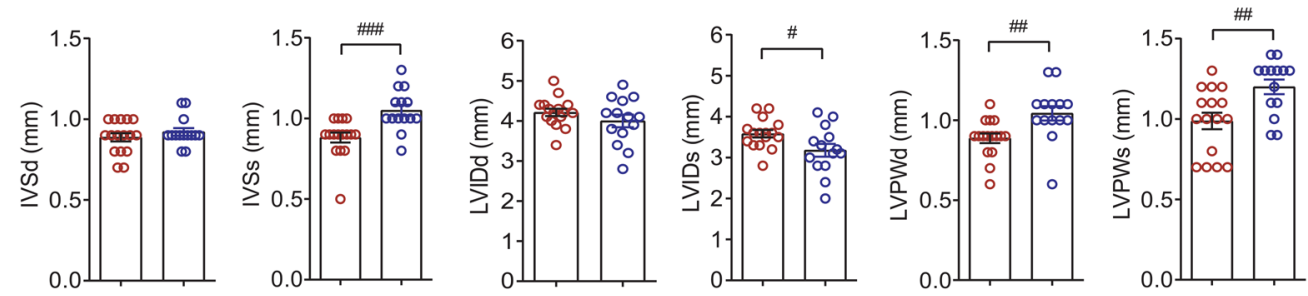

J
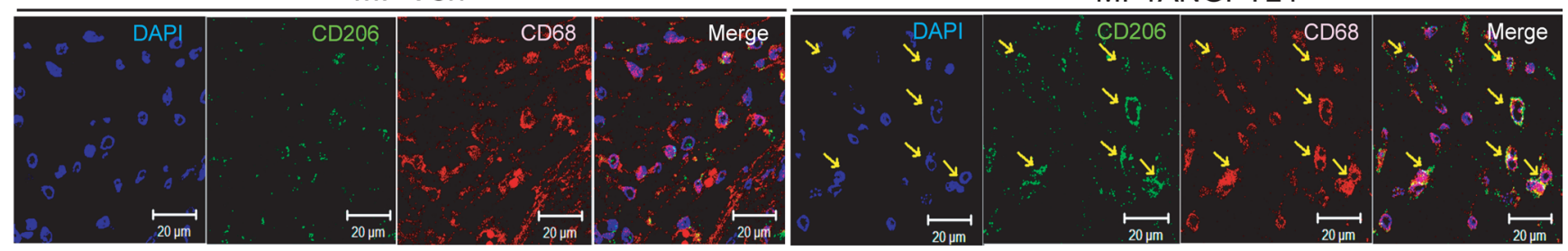
Figure 8. Cardiac inflammation and function are improved by ANGPTL4 treatment in a mouse MI model. (A) Recombinant ANGPTL4 protein, 200 ng, was injected into the mouse MI model. Heart tissues and blood were collected after 2 days. The expression levels of inflammatory markers in the heart tissue were assessed by real-time PCR (B, $n=4)$ and Western blot $(\mathbf{C}, n=3)$. Intensity quantification is representative of mean \pm SEM. (D) Circulating IL-6 $(n=4)$ and IL-1 $\beta(n=5)$ were reduced in ANGPTL4 protein-injected mice. (E) Experimental procedure for analyses of cardiac macrophages at 3 days or 14 days after MI. (F) The CD45+CD11b+F4/80+CD80+ cells were isolated and quantified from heart tissues at 3 days after injections of ANGPTL4 protein. $n=5$. (C) The mRNA levels of Arg1 and Cd206 were assessed in the single cells isolated from heart tissues at 14 days after injections of ANGPTL4 protein or Veh. $n=4$. (H) MI was induced by coronary artery ligation, then 200 ng ANGPTL4 protein was injected i.p. Heart tissues were collected after 14 days. (I) Representative echocardiogram and echocardiographic indexes expressed as graphs. $n=16$ for Veh group; $n=14$ for ANGPTL4 group. (J) Representative confocal images of CD68 ${ }^{+}$macrophages costained with CD206 in the infarcted myocardium. $n=8$ for each group. EF, ejection fraction; FS, fractional shortening; IVSd, intraventricular septal width in diastole; IVSs, intraventricular septal width in systole; LVIDd, left ventricular internal dimension in diastole; LVIDs, left ventricular internal dimension in systole; LVPWd, left ventricular posterior wall thickness in diastole; LVPWs, left ventricular posterior wall thickness in systole. Data are represented as mean \pm SEM. ${ }^{~} P<0.05 ;{ }^{\#} P<0.01 ;{ }^{\# \# P} P<0.001$ (by Student's $t$ test or 1 -way ANOVA with Bonferroni's multiple-comparisons test).

the full promoter construct, and pGL3-1100 promoter lost its activity. These results suggested that the region from -1700 to -2300 is possibly involved in the activation of the ANGPTL4 promoter by inflammatory macrophages (Figure 9I). For confirmatory experiments, we pretreated hMSCs with SR1001 before coculture with THP-1 macrophages and found that SR1001 effectively abolished the inhibition of CXCL10 and CXCL11 induction (Supplemental Figure 8C).

\section{Discussion}

After MSC administration, migration and engraftment of the stem cells in the lesion induce transdifferentiation into functional cells, angiogenesis, wound healing, and the resolution of inflammation. MSCs exhibit remarkable immunosuppressive activities in various diseases $(15,16)$ besides the direct incorporation of MSCs in tissue regeneration, although the mechanisms of these actions remain unresolved.

A number of studies have demonstrated that the therapeutic effects of MSCs are associated with soluble paracrine factors $(6,17,18)$. The antiinflammatory potential of MSCs cannot be pinned to a single factor or mechanism, but rather to soluble factors, many of which are involved in inflammatory environments (18). Macrophages are one of the multiple factors contributing to the pathological progress of cardiovascular disease in terms of stem cell-induced cardiac regeneration and reparative roles of inflammatory responses $(19,20)$.

Critically, the present study identified ANGPTL4 as having an intricate interplay with MSCs against inflammatory macrophages. A screen for molecules whose deficiency blocked the modulatory activity of MSCs against stimulated macrophages pointed to ANGPTL4 as a critical regulator of MSCs, and these in vitro observations were corroborated by in vivo studies in mouse models of peritonitis and MI.

ANGPTL4 belongs to the angiopoietin-like protein (ANGPTL) family, which is structurally similar to the angiopoietins and does not bind to the receptors classically targeted by angiopoietins. The ANGPTL family generally is considered orphan ligands $(21,22)$. Circulating ANGPTL4 is cleaved into an N-terminal fragment and a C-terminal fragment, and mutating the ANGPTL4 cleavage region improves the stability of the full-length protein (23). ANGPTL4 is a paracrine and endocrine regulator of lipid metabolism and is ubiquitously expressed in humans, mainly in the liver, adipose tissue, blood plasma, placenta, small intestine, and heart (24). In MSCs, ANGPTL4 is upregulated to induce MMP-13 during chondrogenic differentiation (25). There have been conflicting reports concerning the role of ANGPTL4 in atherosclerosis. For example, in one study, ANGPTL4 overexpression suppressed lipid overloading in macrophages and reduced atherosclerotic plaque development without hypertriglyceridemia (26). In another study, ANGPTL4-deficient mice had a better lipid profile and smaller atherosclerotic lesions than mice without this deficiency, whereas macrophage-specific deficiency of ANGPTL4 accelerated atherosclerosis progression via vascular inflammation (27). A recent study showed that genetic ablation of ANGPTL4 in adipose tissue resulted in enhanced lipoprotein lipase activity and reduced atherosclerosis (28). These discrepancies can be partially explained by the finding that germline deletion of ANGPTL4 results in severe peritonitis, severe gut inflammation, and decreased survival with a marked reduction in atherosclerosis. Lipoprotein lipase is the primary enzyme that hydrolyzes lipoprotein triglycerides, and ANGPTL4 is the endogenous inhibitor of lipoprotein lipase. A population-based study revealed that low plasma triglyceride levels were significantly related with loss-offunction alleles in ANGPTL4, which is unable to inhibit lipoprotein lipase activity in vitro (29). In nephrotic syndrome, elevated circulating ANGPTL4 protein in podocytes exerts a systemic feedback response against proteinuria and results in hypertriglyceridemia (30). In other studies, carriers of both inactivating mutations 
A

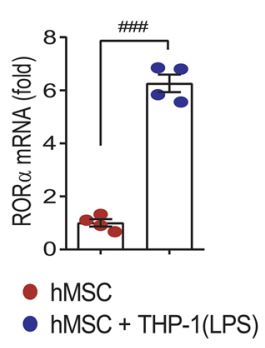

B
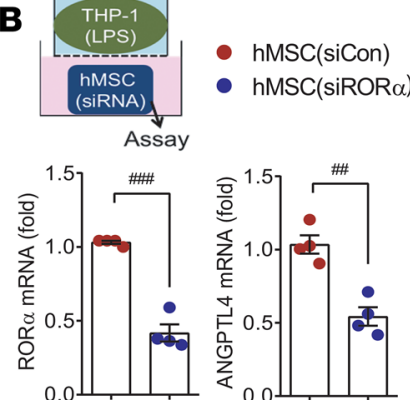

C

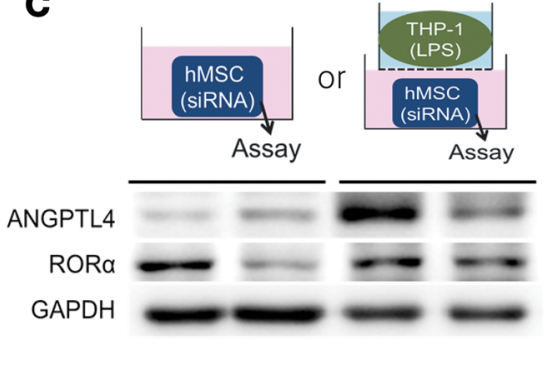

- hBMC(siCon)

- hBMC(siROR $\alpha)$

- $h B M C($ siCon) + THP-1(LPS)

- $\operatorname{hBMC}(\operatorname{siROR} \alpha)+$ THP-1(LPS)

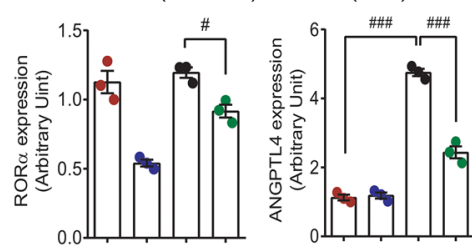

D

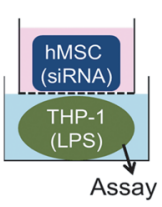

- THP-1(LPS)

- THP-1(LPS) + hMSC(siCon)

- $\mathrm{THP}-1(\mathrm{LPS})+\mathrm{hMSC}(\mathrm{siROR} \alpha)$
E

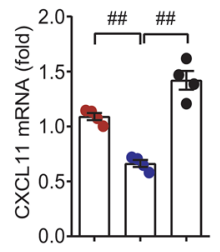

Veh or IL-1 $\beta$ or TNF $\alpha$

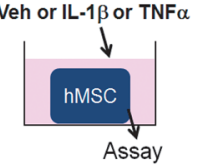

$\mathbf{F}$

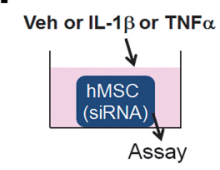

- hMSC(siCon)-Veh

- hMSC(siROR $\alpha)$-Veh

O hMSC(siCon)-IL- $\beta$

O hMSC(siROR $\alpha)$-IL- $\beta$

$\square \mathrm{hMSC}($ siCon)-TNF $\alpha$

$\checkmark \operatorname{hMSC}(\operatorname{siROR} \alpha)-\mathrm{TNF} \alpha$
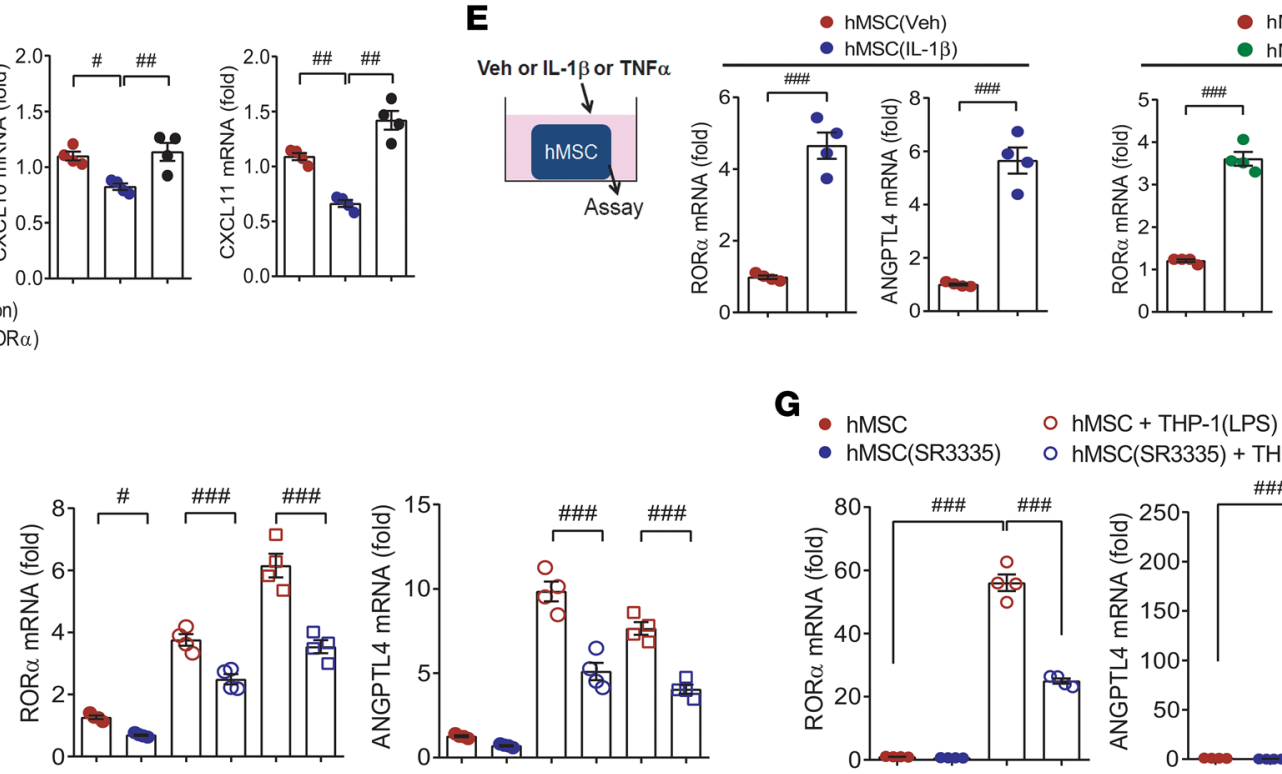

- hMSC(Veh)

$\mathrm{hMSC}(\mathrm{TNF} \alpha)$

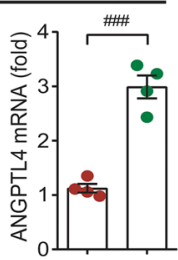

H

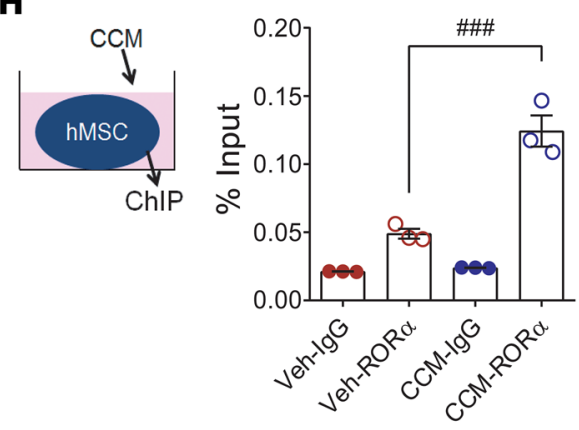

I

G
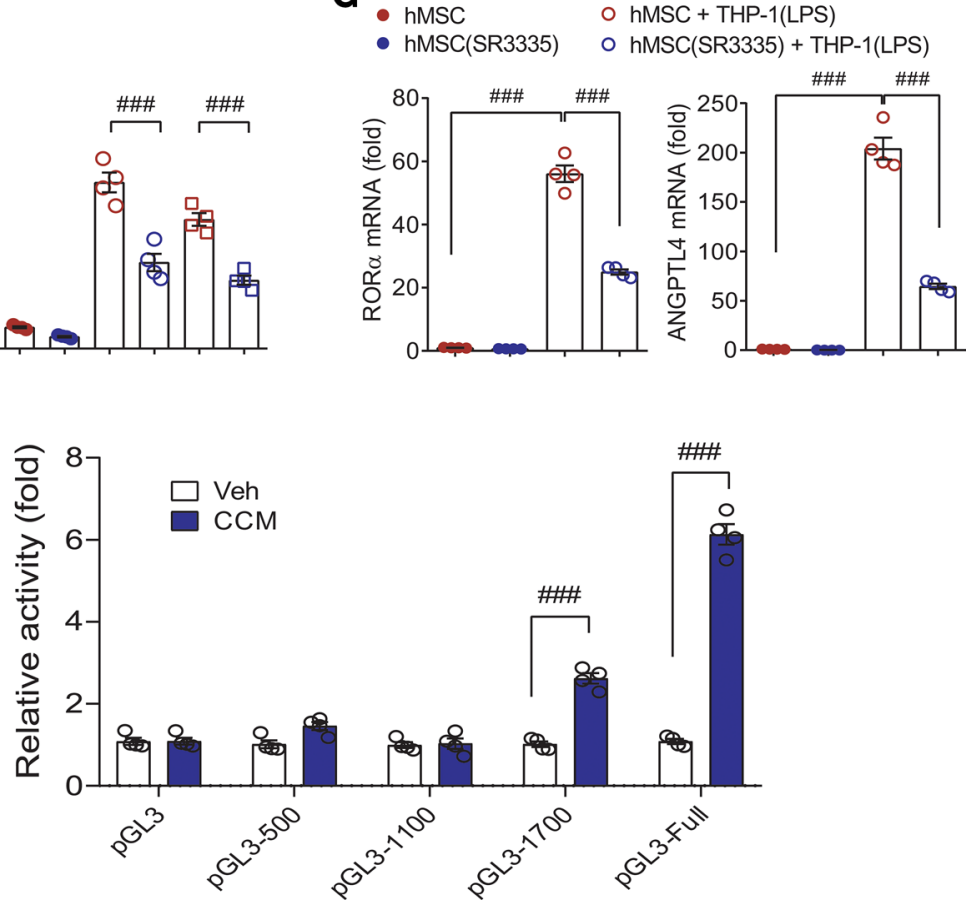

Figure 9. ROR $\alpha$ positively regulates ANGPTL4 induction in MSCs. (A) Rora mRNA expression was assessed in hMSCs with or without coculture with THP-1 macrophages. $n=4$. hMSCs were transfected with control siRNA or ROR $\alpha$ siRNA before coculture with THP-1 macrophages, and expression levels of ROR $\alpha$ and ANGPTL4 mRNA (B, $n=4)$ and protein $(C, n=3)$ were measured in hMSCs. (D) Expression levels of CXCL10 and CXCL11 in macrophages were assayed after coculture with hMSCs transfected with control siRNA or ANGPTL4 siRNA. $n=4$. (E) The mRNA levels of Rora and Angpt/4 were assessed in hMSCs treated with IL-1 $\beta$ or TNF- $\alpha . n=4$. (F) The mRNA levels of Rora and Angpt/4 were assessed in siROR $\alpha$-transfected hMSCs treated with IL-1 $\beta$ or TNF- $\alpha . n=4$. (G) hMSCs were treated with SR1001, an inverse agonist of ROR $\alpha$ and ROR $\gamma$, or SR3335, a selective inverse agonist of ROR $\alpha$, with or without coculture with THP-1 macrophages. Inductions of ROR $\alpha$ and ANGPTL4 were assessed by real-time PCR. $n=4$. (H) hMSCs were stimulated with Veh or CCM, and binding of ROR $\alpha$ to ANGPTL4 promoter was determined by ChIP assay. $n=3$. (I) Deletion analysis of the ANGPTL4 promoter activity. HEK293T cells were transfected with the ANGPTL4-LUC reporter constructs and stimulated with Veh or CCM to analyze promoter activities. $n=4$. Data are represented as mean \pm SEM. ${ }^{\#} P<0.05$; ${ }^{\# \#} P<0.01$; $\# \# \#<0.001$ (by Student's $t$ test or 1-way ANOVA with Bonferroni's multiple-comparisons test). 
in ANGPTL4 and loss-of-function mutations in ANGPTL4 had lower levels of triglycerides, and these mutations were associated with a lower risk of coronary artery disease than in noncarriers $(31,32)$. In a mouse MI model, ANGPTL4 preserved the microcirculatory network and improved endothelial barrier function by restoring vascular integrity during ischemia/reperfusion injury (33). In MI patients, circulating ANGPTL4 levels predict no-reflow after successful percutaneous intervention (34).

ANGPTL4 has multifaceted functions and its physiological roles may be largely dependent on the pathological conditions. In this study, we highlighted that ANGPTL4 was highly induced and released from MSCs in response to inflammatory macrophages. To decipher the role and mechanism of action of ANGPTL4 in MSCs, we first compared the inhibitory effects of naive MSCs and ANGPTL4-deficient MSCs. ANGPTL4 was markedly induced in MSCs in response to coculture with either unstimulated macrophages or LPS-stimulated macrophages (Supplemental Table 1; Figure 1, F-H; and Supplemental Figure 3, B and C). Recombinant ANGPTL4 protein itself substantially inhibited the induction of proinflammatory genes in LPS-stimulated macrophages (Figure 1I), and this finding suggested that ANGPTL4 was specifically induced in MSCs in the inflammatory environment.

Given that ANGPTL4 is substantially induced in MSCs by interaction with macrophages, next we tried to identify an intrinsic inducer of ANGPTL4. Numerous binding sites have been found within the ANGPTL4 promoter, and we selected ROR $\alpha$ as a candidate transcription factor. ROR $\alpha$ is one of the unclear receptors and has been cloned by virtue of its strong homology with the retinoic acid receptor. ROR $\alpha$ binds DNA as a monomer to specific sites called ROR $\alpha$ response elements, which consist of a 6-bp AT-rich sequence preceding the half-core PuGGTCA motif (35). ROR $\alpha$ is a lipid-sensing nuclear receptor with diverse biological functions, including regulation of lipid metabolism and inflammation, and was reported to exert an antiinflammatory role by inhibiting NF-кB p65 translocation (36). We found ROR bound on the ANGPTL4 promoter in MSCs by coculture with macrophages. Genetic and pharmacological disruption of ROR $\alpha$ in MSCs failed to induce ANGPTL4 and inhibited CXCL10 and CXCL11 induction in cocultured macrophages. These results suggested that the ROR $\alpha$-ANGPTL4 axis was an essential component for the immunomodulatory activity of MSCs.

To examine whether administration of CCM to a pathological macrophage-rich environment can ease inflammation, peritonitis and MI were induced in mice. As anticipated, the expression of antiinflammatory phenotype markers in peritoneal macrophages was higher in mice injected with MSC CCM and lower in mice injected with ANGPTL4-knockdown MSC CCM. This in vivo finding was consistent with the in vitro data. More interestingly, application of recombinant ANGPTL4 protein was comparable to MSC CCM in terms of the remarkable suppression of inflammatory macrophages and circulating inflammatory cytokine levels. In addition to peritonitis, we studied the effects of MSC CCM and ANGPTL4 protein in the MI model. The Veh group showed a significant induction of iNOS, whereas no detectable Arg1 was found in the infarcted heart tissue. On the other hand, Arg1 was remarkably increased in the MSC(siCon)-CCM group compared with the MSC(siANGPTL4)-CCM group. Next, we decided to take inspiration from multifunctional stem cells and the ways in which they induce tissue repair and modulate pathological immune cells in inflammatory lesions. Interestingly, proinflammatory mediators were significantly suppressed, and antiinflammatory mediators were upregulated in the infarcted heart tissue by application with recombinant ANGPTL4 protein. The most impressive impact of ANGPTL4 application was the meaningful structural and functional recoveries after MI. The results of this study support the view that a specific pattern of interaction of MSCs with macrophages exists within the disease niche and most likely contributes to the progressive cardiac regeneration and reduction in fibrosis. No previous reports to our knowledge have dealt with the specific role of ANGPTL4 in MSCs from a therapeutic perspective, and our results demonstrate that ANGPTL4 delivery is efficacious both for easing inflammation as well as for inducing tissue regeneration.

Transplantation of MSCs has several potential disadvantages. The engraftment rate is low, DNA can be damaged during in vitro expansion, and the therapeutic effect is dependent on cell dose, delivery route, and timing. Several reports have shown that some patients who receive adult stem cell therapy occasionally have unexpected adverse outcomes, such as brain tumor (37), angiomyeloproliferative lesions (38), and glioproliferative lesions (39). A recent study identified missense mutations in TP53, a tumor suppressor, in both human embryonic stem cells and human induced pluripotent stem cells, and suggested the need for regular genetic testing of stem cells for further clinical applications (40). Current issues concerning stem cell therapy that should be aggressively considered include the lack of long-term follow-up of the cell fate of transplanted stem cells, possible involvement in tumor formation, and undisclosed mutations (38-41). Translation of basic 
research to clinical trials can hardly succeed because of these issues, and thus the use of an MSC-derived soluble factor, such as ANGPTL4, could be a better and safer opportunity for cell-free regeneration therapy. Instead of intensive concentration on developing techniques to increase the therapeutic efficacy of stem cells, replacing stem cells with an apparent effector molecule would be a better choice for safe and effective therapy.

Here we have demonstrated the potentially novel antiinflammatory role of ANGPTL4 secreted from MSCs in a pathological microenvironment, and we define a molecular mechanism by which ROR $\alpha$ /ANGPTL4 regulates inflammation-related gene expression in macrophages (Supplemental Figure 9). Our further work will specifically address the role of ANGPTL4 following MI in ANGPTL4-deficient mice.

\section{Methods}

All supporting data are available within the article and the Supplemental Methods.

We studied the role of ANGPTL4 in isolated MSCs and in a MI mouse model. Mice underwent permanent left coronary artery occlusion protocols. The detailed methodology is provided in the Supplemental Methods.

Mice. Male inbred BALB/c nude mice were purchased from Orient Bio Inc. Angptl4-knockout mice on a C57BL/6J genetic background were provided by Min-Seon Kim (University of Ulsan College of Medicine, Seoul, Korea) (42).

Cell culture. hMSCs, immortalized by the introduction of telomerase, were provided by Yeon-Soo Kim (Inje University, Seoul, South Korea). hATSCs were obtained in accordance with guidelines approved by Chonnam National University Hospital Institutional Review Board (CNUH-2016-266). mMSCs were isolated from bone marrow and cultured (7). Bone marrow cells from mice were collected by flushing the femurs and tibias and were cultured, and the mMSCs were used within 4 passages.

Human macrophages were differentiated from $\mathrm{CD}_{1} 4^{+}$monocytes isolated from peripheral blood. Blood sampling was performed under protocols approved by the Institutional Review Board of Chonnam National University Hospital (CNUHH-2018-018). Peripheral blood mononuclear cells were isolated, and $\mathrm{CD} 14^{+}$monocytes were separated by using anti-CD14 mAb-coupled magnetic beads (CD14 MicroBeads; Miltenyi Biotech, 130-050-201) followed by MACS column separation (43). Monocytes were treated with GM-CSF and differentiated into macrophages with LPS, IFN- $\gamma$, and IL-6 (44). Human monocytic THP-1 cells, purchased from the Korean Cell Lin Bank, were differentiated into macrophages by PMA (MilliporeSigma, 8139, $200 \mathrm{nmol} / \mathrm{L}$ ) treatment. For BMDMs, mononuclear cells were isolated from mouse bone marrow and cultured for 7 days in macrophage differentiation medium (supplemented with 30\% L929 cell-conditioned medium, 20\% fetal bovine serum [Gibco, 16000044], and 50\% RPMI1640 [Gibco, 11875-093]). BMDMs were stimulated with LPS 100 ng/mL (MilliporeSigma, L4391) and IFN- $\gamma 30 \mathrm{ng} / \mathrm{mL}$ (Thermo Fisher Scientific, PHC4031) for 24 hours.

HEK293T cells were cultured in Dulbecco's modified Eagle's medium (Gibco, Thermo Fisher Scientific, 11965-092) supplemented with 10\% fetal bovine serum and penicillin/streptomycin.

Isolation of neonatal rat cardiomyocytes and cardiac fibroblasts. Primary cardiomyocytes and cardiac fibroblasts were isolated from 2-day-old Sprague-Dawley rats as described in a previous study (10).

Reagents and plasmids. The reagents and plasmids used in this study are described in the Supplemental Methods. Mouse primers are listed in Supplemental Table 2, and the antibodies are listed in Supplemental Table 3.

Statistics. All data are expressed as mean \pm SEM from at least 3 independent experiments. Differences between experimental and control groups were analyzed by 2-tailed Student's $t$ test or 1-way ANOVA with Bonferroni's multiple-comparisons test using SPSS. A $P$ value less than 0.05 was considered significant.

Study approval. All mouse experiments were performed after approval by our local ethical committee at Chonnam National University Medical School (CNU IACUC-H-2016-37). hATSCs were obtained in accordance with guidelines approved by Chonnam National University Hospital Institutional Review Board (CNUH-2016-266). Blood sampling was performed under protocols approved by the Institutional Review Board of Chonnam National University Hospital (CNUHH-2018-018).

\section{Author contributions}

YSK, YA, and DIC designed the research studies, analyzed the results, and wrote the manuscript. DIC, HJK, JHJ, GHE, HHC, MHH, MRK, MC, HYJ, and HCC performed the experiments and prepared the figures. 


\section{Acknowledgments}

We would like to acknowledge Yeon-Soo Kim for providing immortalized human bone marrow-derived MSCs. This work was supported by the National Research Foundation of Korea (NRF-2015M3A9B4066496, 2015M3A9C6031684, 2016R1D1A1A09917796, 2018R1D1A1B07040813), a grant of the Korea Health Technology R\&D Project through the Korea Health Industry Development Institute (funded by the Ministry of Health \& Welfare, Republic of Korea, HI18C1352), and a grant (BCRI18017) of Chonnam National University Hospital Biomedical Research Institute.

Address correspondence to: Youngkeun Ahn, Cell Regeneration Research Center, Department of Cardiology, Chonnam National University Hospital, 42 Jebong-ro, Dong-gu, Gwangju, 61469, Korea. Phone: 82.62.220.4764; Email: cecilyk@hanmail.net. Or to: Yong Sook Kim, Cell Regeneration Research Center, Biomedical Research Institute, Chonnam National University Hospital, 42 Jebong-ro, Dong-gu, Gwangju, 61469, Korea. Phone: 82.62.220.6188; Email: reorgan@hanmail.net.

1. Loffredo FS, Steinhauser ML, Gannon J, Lee RT. Bone marrow-derived cell therapy stimulates endogenous cardiomyocyte progenitors and promotes cardiac repair. Cell Stem Cell. 2011;8(4):389-398.

2. Cho DI, et al. The optimization of cell therapy by combinational application with apicidin-treated mesenchymal stem cells after myocardial infarction. Oncotarget. 2017;8(27):44281-44294.

3. Teerlink JR, et al. Benefit of cardiopoietic mesenchymal stem cell therapy on left ventricular remodelling: results from the Congestive Heart Failure Cardiopoietic Regenerative Therapy (CHART-1) study. Eur J Heart Fail. 2017;19(11):1520-1529.

4. Kanelidis AJ, Premer C, Lopez J, Balkan W, Hare JM. Route of delivery modulates the efficacy of mesenchymal stem cell therapy for myocardial infarction: a meta-analysis of preclinical studies and clinical trials. Circ Res. 2017;120(7):1139-1150.

5. Goh YY, et al. Angiopoietin-like 4 interacts with integrins beta1 and beta5 to modulate keratinocyte migration. Am J Pathol. 2010;177(6):2791-2803.

6. Timmers L, et al. Human mesenchymal stem cell-conditioned medium improves cardiac function following myocardial infarc tion. Stem Cell Res. 2011;6(3):206-214.

7. Cho DI, et al. Mesenchymal stem cells reciprocally regulate the M1/M2 balance in mouse bone marrow-derived macrophages Exp Mol Med. 2014;46:e70.

8. Kim YS, et al. Protective role of 5-azacytidine on myocardial infarction is associated with modulation of macrophage phenotype and inhibition of fibrosis. J Cell Mol Med. 2014;18(6):1018-1027.

9. Jeong HY, et al. 5-Azacytidine modulates interferon regulatory factor 1 in macrophages to exert a cardioprotective effect. Sci Rep. 2015;5:15768.

10. Kim YS, et al. Natural product derivative BIO promotes recovery after myocardial infarction via unique modulation of the cardiac microenvironment. Sci Rep. 2016;6:30726.

11. Gomez Perdiguero E, et al. ANGPTL4- $\alpha \mathrm{v} \beta 3$ interaction counteracts hypoxia-induced vascular permeability by modulating Src signalling downstream of vascular endothelial growth factor receptor 2. J Pathol. 2016;240(4):461-471.

12. Bouzegrhane F, Thibault G. Is angiotensin II a proliferative factor of cardiac fibroblasts? Cardiovasc Res. 2002;53(2):304-312.

13. Beuscher HU, Günther C, Röllinghoff M. IL-1 beta is secreted by activated murine macrophages as biologically inactive precursor. J Immunol. 1990;144(6):2179-2183.

14. Stow JL, Murray RZ. Intracellular trafficking and secretion of inflammatory cytokines. Cytokine Growth Factor Rev. 2013;24(3):227-239.

15. Spaggiari GM, Capobianco A, Abdelrazik H, Becchetti F, Mingari MC, Moretta L. Mesenchymal stem cells inhibit natural killer-cell proliferation, cytotoxicity, and cytokine production: role of indoleamine 2,3-dioxygenase and prostaglandin E2. Blood. 2008;111(3):1327-1333.

16. Duffy MM, Ritter T, Ceredig R, Griffin MD. Mesenchymal stem cell effects on T-cell effector pathways. Stem Cell Res Ther. 2011;2(4):34.

17. Uemura R, Xu M, Ahmad N, Ashraf M. Bone marrow stem cells prevent left ventricular remodeling of ischemic heart through paracrine signaling. Circ Res. 2006;98(11):1414-1421.

18. Ranganath SH, Levy O, Inamdar MS, Karp JM. Harnessing the mesenchymal stem cell secretome for the treatment of cardiovascular disease. Cell Stem Cell. 2012;10(3):244-258.

19. Ben-Mordechai T, et al. Macrophage subpopulations are essential for infarct repair with and without stem cell therapy. $J$ Am Coll Cardiol. 2013;62(20):1890-1901.

20. Aurora AB, et al. Macrophages are required for neonatal heart regeneration. J Clin Invest. 2014;124(3):1382-1392.

21. Mandard S, et al. The fasting-induced adipose factor/angiopoietin-like protein 4 is physically associated with lipoproteins and governs plasma lipid levels and adiposity. J Biol Chem. 2006;281(2):934-944.

22. Yoshida K, Shimizugawa T, Ono M, Furukawa H. Angiopoietin-like protein 4 is a potent hyperlipidemia-inducing factor in mice and inhibitor of lipoprotein lipase. J Lipid Res. 2002;43(11):1770-1772.

23. Yin W, Romeo S, Chang S, Grishin NV, Hobbs HH, Cohen JC. Genetic variation in ANGPTL4 provides insights into protein processing and function. J Biol Chem. 2009;284(19):13213-13222.

24. Dijk W, Kersten S. Regulation of lipoprotein lipase by Angpt14. Trends Endocrinol Metab. 2014;25(3):146-155.

25. Mathieu M, et al. Involvement of angiopoietin-like 4 in matrix remodeling during chondrogenic differentiation of mesenchymal stem cells. J Biol Chem. 2014;289(12):8402-8412.

26. Georgiadi A, et al. Overexpression of angiopoietin-like protein 4 protects against atherosclerosis development. Arterioscler 
Thromb Vasc Biol. 2013;33(7):1529-1537.

27. Aryal B, et al. ANGPTL4 deficiency in haematopoietic cells promotes monocyte expansion and atherosclerosis progression. Nat Commun. 2016;7:12313

28. Aryal B, et al. Absence of ANGPTL4 in adipose tissue improves glucose tolerance and attenuates atherogenesis. JCI Insight. 2018;3(6):97918.

29. Romeo S, et al. Population-based resequencing of ANGPTL4 uncovers variations that reduce triglycerides and increase HDL. Nat Genet. 2007;39(4):513-516.

30. Clement LC, Macé C, Avila-Casado C, Joles JA, Kersten S, Chugh SS. Circulating angiopoietin-like 4 links proteinuria with hypertriglyceridemia in nephrotic syndrome. Nat Med. 2014;20(1):37-46.

31. Dewey FE, et al. Inactivating Variants in ANGPTL4 and Risk of Coronary Artery Disease. NEngl J Med. 2016;374(12):1123-1133.

32. Myocardial Infarction Genetics CARDIoGRAM Exome Consortia Investigators, et al. Coding variation in ANGPTL4, LPL, and SVEP1 and the risk of coronary disease. N Engl J Med. 2016;374(12):1134-1144.

33. Galaup A, et al. Protection against myocardial infarction and no-reflow through preservation of vascular integrity by angiopoietin-like 4. Circulation. 2012;125(1):140-149.

34. Bouleti C, et al. Angiopoietin-like 4 serum levels on admission for acute myocardial infarction are associated with no-reflow. Int J Cardiol. 2015;187:511-516.

35. Gawlas K, Stunnenberg HG. Differential binding and transcriptional behaviour of two highly related orphan receptors, ROR alpha(4) and ROR beta(1). Biochim Biophys Acta. 2000;1494(3):236-241.

36. Delerive $\mathrm{P}$, et al. The orphan nuclear receptor ROR alpha is a negative regulator of the inflammatory response. EMBO Rep. 2001;2(1):42-48.

37. Amariglio N, et al. Donor-derived brain tumor following neural stem cell transplantation in an ataxia telangiectasia patient. PLoS Med. 2009;6(2):e1000029.

38. Thirabanjasak D, Tantiwongse K, Thorner PS. Angiomyeloproliferative lesions following autologous stem cell therapy. $J$ Am Soc Nephrol. 2010;21(7):1218-1222.

39. Kridin K, Bergman R, Khamaisi M, Zelber-Sagi S, Weltfriend S. Cement-Induced chromate occupational allergic contact dermatitis. Dermatitis. 2016;27(4):208-214.

40. Merkle FT, et al. Human pluripotent stem cells recurrently acquire and expand dominant negative P53 mutations. Nature. 2017;545(7653):229-233.

41. Coombs CC, et al. Therapy-Related clonal hematopoiesis in patients with non-hematologic cancers is common and associated with adverse clinical outcomes. Cell Stem Cell. 2017;21(3):374-382.e4.

42. Kim HK, et al. Hypothalamic Angpt14/Fiaf is a novel regulator of food intake and body weight. Diabetes. 2010;59(11):2772-2780.

43. Bhattacharjee J, Das B, Mishra A, Sahay P, Upadhyay P. Monocytes isolated by positive and negative magnetic sorting techniques show different molecular characteristics and immunophenotypic behaviour. F1000Res. 2017;6:2045.

44. Zarif JC, Hernandez JR, Verdone JE, Campbell SP, Drake CG, Pienta KJ. A phased strategy to differentiate human CD14+monocytes into classically and alternatively activated macrophages and dendritic cells. BioTechniques. 2016;61(1):33-41. 